\title{
Three Conditions Leading to a Unified (Quasi-Newtonian) Physics
}

\author{
Alfredo Bacchieri \\ University of Bologna, Bologna, Italy \\ Email: abacchieri@libero.it
}

How to cite this paper: Bacchieri, A. (2018) Three Conditions Leading to a Unified (Quasi-Newtonian) Physics. Journal of Modern Physics, 9, 2045-2072.

https://doi.org/10.4236/jmp.2018.911129

Received: June 28, 2018

Accepted: September 11, 2018

Published: September 14, 2018

Copyright $\odot 2018$ by author and Scientific Research Publishing Inc. This work is licensed under the Creative Commons Attribution International License (CC BY 4.0).

http://creativecommons.org/licenses/by/4.0/

\begin{abstract}
It is shown that the total escape speed $u$ (i.e. from all the masses in space), which depends on the total gravitational potential $U$ through the relation $u=$ $(-2 U)^{1 / 2}$, tends to $c$, then, under the $1^{\text {st }}$ condition $c=u$, and assuming (as a $2^{\text {nd }}$ condition) the light as composed of longitudinally-extended, elastic (i.e. variable length) and massive particles, (photons), emitted at speed $u$ referred to the initial location $(\mathrm{O})$ of their source, we show that $c$ referred to $\mathrm{O}$ becomes invariant despite any motion of its source from O. We revised the Doppler effect for the light, the gravitational redshift cause, the time dilation, highlighting the differences with respect to the Relativity. In the $2^{\text {nd }}$ part, considering ( $3^{\text {rd }}$ condition) the electron charge as a point-particle fixed to the electron surface and facing the atom nucleus during the electron orbit, the light-matter interaction becomes a consequence of the particular impacts between these photons and the circling electrons: e.g., on $\mathrm{H}$ atom, we found 137 circular orbits only, the last one being the ionization orbit, where the electron orbital speed becomes $v_{i}=c / 137^{2}$. [Classical physics, under the assumption that a circling electron should produce (like a macroscopic electric circuit), an electro-magnetic radiation, implies that this claimed effect has to cause the electron fall into its nucleus: on Section 2.5, we show that the e.m. radiation of a circling electron only happens between two circular orbits].
\end{abstract}

\section{Keywords}

Doppler Effect for the Light, Harvard Tower Experiment, Gravitational Redshift, Time Dilation, Absorption/Emission, Photoelectric and Compton Effects

\section{Speed of Light}

\subsection{Introduction}

This paper is based on the following assumptions: 
1) Finite mass of the universe.

2) Newton's absolute time and space.

Then, the following two conditions:

- Equality between the speed of light $c$ and the total escape speed $u$.

- Light composed of longitudinal-extended, elastic particles, as defined on Section 1.4

We obtain, on the $1^{\text {st }}$ Part, these main results:

1) The measured constancy of $c$ is due, first, to the constancy of the total gravitational potential $U$ on Earth: indeed, the max variation of $U$ on Earth, occurring between Aphelion and Perihelion, leads to a negligible $\Delta u_{\mathrm{AP}}=0.10 \mathrm{~m} \cdot \mathrm{s}^{-1}$; moreover, the speed of light emitted by a source, either fixed or in motion from its initial location $\mathrm{O}$ (where the photons emission starts), is invariant with respect to $\mathrm{O}$;

2) Doppler effect equations for the light, slightly different from the relativistic ones;

3) The compensating velocity, (to restore the resonance source-absorber on Harvard Tower Experiment), has same value but contrary direction with respect to the one predicted by GR;

4) The variation of $U$ from ground to GPS satellite level, implying a decrease of $c$ and consequently a decrease of the frequency of the photons emitted by atomic clocks at that altitude, give them an increase of their counted time (inducing them to run faster);

5) High gravitational redshifts are due to the increase of $c$ (inducing the increase of the photons length $\lambda$ ) during their path toward the Earth where $\left|U_{\mathrm{o}}\right|>>$ $\left|U_{\rightarrow \infty}\right|$.

On the $2^{\text {nd }}$ part, under the third condition claiming that the electron charge can be considered as a point-particle fixed to the electron surface, and facing the atom nucleus during the electron orbit, we got these results:

$\mathrm{On} \mathrm{H}$ atom:

6) $n=1,2, \cdots, 137$ electron circular progressive orbits, not an infinite number; $n$ also represents the number of admitted photons (of the same ray) absorbed (or emitted) by the electron during two electron orbits: (in fact, the photons total number along each circular double-orbit (in short d-orbit) becomes $n^{2}$, as shown on Section 2.4);

7) The electron radius becomes $r_{\mathrm{e}}=[(1 / 137 \alpha)-1] r_{0}$ (instead of its classical value $r_{\mathrm{e}}=\alpha^{2} r_{\mathrm{B}}$ with $r_{\mathrm{B}}=\alpha / 4 \pi R_{\infty}$ the Bohr radius and $\alpha$ the fine structure constant); $r_{0}=\alpha / 4 \pi R_{\mathrm{H}}$ is the orbit of the electron charge, while the orbit of the electron barycenter (electron effective orbit) becomes $r_{0}^{\prime}=r_{0}+r_{\mathrm{e}}=r_{0} / 137 \alpha$;

8) The electron charge orbital speed along $r_{0}$ becomes $v_{0}=\alpha c$, while the electron effective orbital speed along the orbit $r_{0}^{\prime}$ becomes $v_{0}^{\prime}=c / 137$;

9) The electron radial speed (due to the impacts photons-electron) becomes $w_{n^{2}}=c / 137^{2}$ constant for every circular orbit; along the orbit \#137, the electron orbital speed becomes $v_{137}=c / 137^{2}$ and since $w_{n^{2}}=v_{137}$ there is ionization. 
10) On Photoelectric effect the number of photons hitting a circling electron varies from $n_{\mathrm{f}}$ related to the specific threshold frequency $v_{\mathrm{f}}\left(=W_{\mathrm{f}} / h\right)$ to $\left(n_{\mathrm{f}}\right)^{1 / 2}$ related to a higher frequency corresponding to the lower frequency occurring for the Compton effect; for instance, it is shown that, as for Caesium (having $W_{\mathrm{f}} \cong 2$ $\mathrm{eV}), n_{\mathrm{f}}=361$;

11) Compton effect (CE) needs one photon only; our Doppler effect equations for the light lead to Compton Eq., while, as for the Relativity, the CE is not a Doppler effect.

\subsection{Total Escape Speed $u$ and Evidences That $u=c$}

As known, considering in space only one mass $M$, (for simplicity its barycenter), the gravitational potential $U$ in a point $\mathrm{O}$, assuming $U_{\infty}=0$, is $U=-M G / s$, with $s$ the distance $M$-O. The conservation of energy applied to a particle $m$ (of unitary mass) coming from the infinity, gives $E=U+K$, with $K=1 / 2 u^{2}$ its kinetic energy, therefore for $E=0$, one finds $U=-K$, yielding

$$
u=\sqrt{-2 U}=\sqrt{2 M G / s}
$$

which is a scalar, (called escape speed), representing the value of the velocity $\mathbf{u}$, (referred to $M$ ), a massive particle needs to reach the infinity; hence the relation

$$
\mathbf{v}_{\mathrm{Mm}}=\mathbf{u} \quad\left[\text { with } \quad|\mathbf{u}|=u=(-2 U)^{1 / 2}\right]
$$

represents the condition, as for a point-particle $m$, to be provided with the absolute escape velocity $\mathbf{u}$ (absolute means here referred to $M$ ) whereas considering a generic point $\mathrm{O}$,

$$
\mathbf{v}_{\mathrm{Om}} \equiv \mathbf{u}_{\mathrm{O}} \quad\left[\text { with } \quad\left|\mathbf{u}_{\mathrm{O}}\right|=u_{\mathrm{O}}=\left(-2 U_{\mathrm{O}}\right)^{1 / 2}\right]
$$

represents the condition, regarding $m$, to be provided with the relative escape velocity $\mathbf{u}_{\mathrm{O}}$, where relative means here referred to a generic point $\mathrm{O}$; (anyhow, our claimed variable length of photons, during their emission from a source $S$ gives them an invariant speed referred to any considered point $O$, in spite of any velocity $\mathbf{v}_{\mathrm{OS}}$ ).

Regarding the possibility to define the escape speed from two or more masses, we point out that considering $M$ as a real mass, the potential $U$, in a considered point, has to be regarded as the sum of the partial potentials $U_{n}$ due to each partial mass $M_{n}$ composing the mass $M$; therefore, as for two masses, we have

$$
U_{1,2} \equiv U_{1}+U_{2}=-\left(M_{1} G / s_{1}\right)-\left(M_{2} G / s_{2}\right)
$$

yielding $u_{1,2} \equiv \sqrt{-2 U_{1,2}}=\sqrt{-2\left(U_{1}+U_{2}\right)}$ thus we can write

$$
u_{1,2}^{2}=-2 U_{1,2}=u_{1}^{2}+u_{2}^{2}
$$

while for all the $n$ masses in space we get

$$
u \equiv(-2 U)^{1 / 2}=\left(-2 \Sigma U_{n}\right)^{1 / 2}=\left(2 \Sigma M_{n} G / s_{n}\right)^{1 / 2}=\sqrt{\Sigma u_{n}^{2}}
$$

representing the total escape speed.

For instance, the escape speed from the Earth surface, due to Earth and Sun only, becomes $u_{\mathrm{E}, \mathrm{S}}^{2}=u_{\mathrm{E}}^{2}+u_{\mathrm{S}}^{2}=\left(2 M_{\mathrm{E}} G / R_{\mathrm{E}}\right)+\left(2 M_{\mathrm{S}} G / d\right)$ with $R_{\mathrm{E}}$ the Earth ra- 
dius, and $d$ the distance Earth-Sun giving $u_{\mathrm{E}, \mathrm{S}} \cong 42 \mathrm{~km} / \mathrm{s}$, while the escape speed from our Galaxy can be roughly expressed as $u_{\mathrm{g}} \simeq\left(2 M_{\mathrm{g}} G / d_{\mathrm{g}}\right)^{1 / 2}$ where $M_{\mathrm{g}} \simeq$ $10^{42} \mathrm{~kg}$ is our Galaxy mass and $d_{\mathrm{g}} \simeq 30 \mathrm{kly} \simeq 3 \times 10^{20} \mathrm{~m}$ the distance between the Earth and our Galaxy centre, giving $u_{\mathrm{g}} \simeq 8 \times 10^{5} \mathrm{~m} \cdot \mathrm{s}^{-1}$.

Now, the mass of universe, by some authors, is estimated [1] [2] [3] to be $M_{\mathrm{u}}$ $\cong 10^{53} \mathrm{~kg}$; about the same value is given through the number $\left(\cong 10^{22}\right)$ of observable stars [4] [5] [6], and since from Earth the distribution of the masses appears to be homogeneous and isotropic, under our assumption $U_{\infty}=0$, we may assume their density as decreasing toward the infinity like a function $\rho=\rho_{\mathrm{c}} \mathrm{e}^{-a s}$ being $\rho_{\mathrm{c}} \cong 9.2 \times 10^{-27} \mathrm{~kg} / \mathrm{m}^{3}$ the critical density.

Writing the mass of universe as

$$
M_{\mathrm{u}}=\int_{0}^{\infty} 4 \pi s^{2} \rho_{c} \mathrm{e}^{-a s} \mathrm{~d} s=4 \pi \rho_{c} \int_{0}^{\infty} s^{2} \mathrm{e}^{-a s} \mathrm{~d} s=\frac{8 \pi \rho_{c}}{a^{3}} \cong 10^{53} \mathrm{~kg}
$$

we find

$$
a=\left(8 \pi \rho_{c} / M_{\mathrm{u}}\right)^{\frac{1}{3}} \cong 1.3 \times 10^{-26} \mathrm{~m}^{-1}
$$

and since, on Earth, the variation of potential due to an increase of the distance $\mathrm{d} s$, can be written $\mathrm{d} U=-\mathrm{d} m G / s$ with $\mathrm{d} m=\rho 4 \pi s^{2} \mathrm{~d} s$ and $\rho=\rho_{\mathrm{c}} \mathrm{e}^{-a s}$, the potential on Earth becomes

$$
\begin{aligned}
U_{0} & =-\int_{0}^{\infty}\left(4 \pi s^{2} / s\right) G \rho_{c} \mathrm{e}^{-a s} \mathrm{~d} s=-4 \pi \rho_{c} G \int_{0}^{\infty} \mathrm{e}^{-a s} s \mathrm{~d} s \\
& =-4 \pi \rho_{c} G / a^{2} \cong-4.5 \times 10^{16} \mathrm{~J}
\end{aligned}
$$

yielding

$$
u_{0}=\left(-2 U_{0}\right)^{1 / 2} \cong\left(9 \times 10^{16}\right)^{1 / 2} \cong 3 \times 10^{8} \mathrm{~m} / \mathrm{s} .
$$

We can therefore infer that, on Earth, $u_{0}=c_{0}$, giving $c_{0}^{2} / 2=-U_{0}$ and, in general,

$$
c=(-2 U)^{1 / 2}=u .
$$

The equality $c=u$, which implies the massiveness of light, means that, along any free path, the speed of light only depends on the value of the potential along that path.

The equality $c=u$ is also supported by a cosmological reason: in fact, if $c>u$ the energy of light will be lost forever and furthermore the observable masses, following the always increasing mass of light going toward the infinity, will also tend to the infinity moving away from each other. On the contrary, if $c<u$, all the masses in space, (having speed lower than $u$ ), will tend to a gravitational collapse, whereas for $c=u$, the mass of light, tending to the infinity in an unlimited time, appears to be the necessary mass to avoid the two events (collapse or dispersion).

\subsection{Annual Variation, on Earth, of the Total Escape Speed}

On Earth, a small variation of the total escape speed $u_{0}$, from Equation (11) written as $u^{2}=-2 U$ yielding $2 u=-2 \mathrm{~d} U / \mathrm{d} u$, can be written as 


$$
\Delta u=-\Delta U / u_{0}
$$

where $\Delta U$ is the variation of the total potential on Earth, mainly due to the variable distance Earth-Sun; so, between Aphelion and Perihelion, where $a=(1+$ $e) d$ and $p=(1-e) d$ are the respective distances Earth-Sun, with $e(=0.0167)$ the eccentricity of the Earth's orbit and $d \cong 1.5 \times 10^{11} \mathrm{~m}$ the average distance Earth-Sun, and since $p a \cong d^{2}$, and $(a-p)=2 e d$, we get

$$
\begin{aligned}
\Delta u_{\mathrm{AP}} & =-\Delta U_{\mathrm{AP}} / u_{0}=-\left(U_{\mathrm{P}}-U_{\mathrm{A}}\right) / u_{0}=\left[\left(M_{\mathrm{S}} G / p\right)-\left(M_{\mathrm{S}} G / a\right)\right] / u_{0} \\
& \cong M_{\mathrm{S}} G(a-p) / d^{2} u_{0} \cong M_{\mathrm{S}} G 2 e d / d^{2} u_{0} \cong M_{\mathrm{S}} G e / d u_{0} \cong+0.10 \mathrm{~m} \mathrm{~s}^{-1}
\end{aligned}
$$

with $M_{\mathrm{S}}=2 \times 10^{30} \mathrm{~kg}$ the mass of the Sun, and we note that $\Delta u_{\mathrm{AP}}$ is compatible with the accuracy of the value of the speed of light in vacuum (on Earth) $c_{0}=$ $299,792,458 \mathrm{~m} \cdot \mathrm{s}^{-1}$.

\subsection{Definition of Photons and Their Speed}

The Galileo's velocity composition law (since different parts of a moving object may have different velocity) is strictly valid for point-particles, and therefore the light cannot be a succession of point-particles (otherwise it should follow this Galileo's law), hence we may infer that the light, to comply both this law and the equality $c=u$, could be composed of particular particles, photons, (whose physical characteristics are then shown on $\$ 2.2$ ), as defined:

Longitudinally-extended, elastic (variable length) and massive particles emitted by a source during an emission time $T$ at speed $c=u$ moving individually or along rays, (continuous succession of photons where every tail corresponds to the front of the next one).

Photons speed: the measurement of the speed of a point-particle (or regarding an object, its barycentre), is defined through the ratio $v=d / t$ with $d$ the distance between two Observers $\left(\mathrm{O}_{1}\right.$ and $\left.\mathrm{O}_{2}\right)$ and $t\left(=t_{2}-t_{1}\right)$ the time the particle/barycentre needs to cover such a distance; on the contrary, every photon may have different length with respect to two Observers, (like, for instance, an elastic thread during its stretching), hence to define the speed of one photon, we must consider its length $\lambda$ divided its transit time $T_{t}$ (time the whole particle needs to cross one Observer). Therefore the relation

$$
c=\lambda / T_{t}
$$

represents the speed of one photon referred to a considered Observer, whereas the relation $c=d / t$, written as

$$
c=d / t=n \lambda / n T_{t}=\lambda / T_{t}
$$

where $n$ is the number of photons (of one ray) each of them having length $\lambda$, and where $t=n T_{t}$ is the transit time of the $n$ photons, represents the average speed of the light along $d$.

\subsection{Invariance of $c$ for Observers at Rest with Respect to the Initial Location $\mathbf{O}$ of a Source of Light, Despite Any Velocity $v_{0 S}$}

Referring to Figure 1, let $\mathrm{O}$ be the location (with respect to all the masses in 


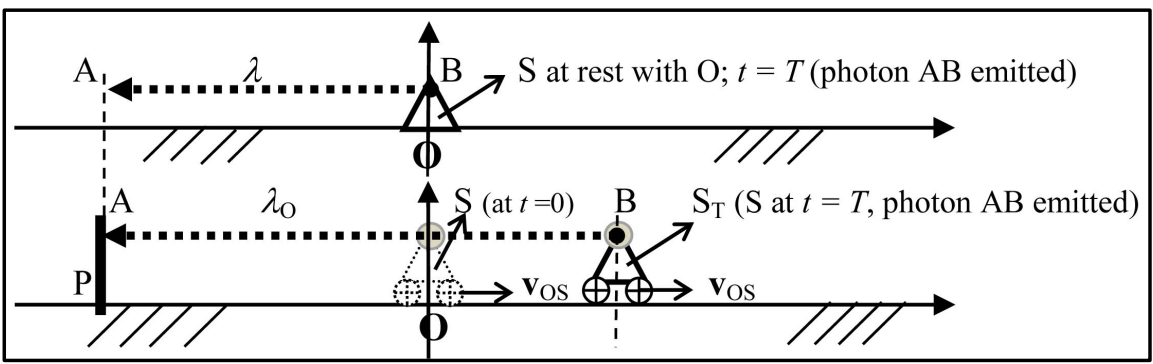

Figure 1. Emission of the photon $A B$ by a source $S$ either at rest or in motion from $\mathrm{O}$.

space) of the source $\mathrm{S}$ at the time $(t=0)$ the front $\mathrm{A}$ is emitted; therefore $\mathrm{O}$ represents the location of the initial emission of a considered photon.

The Earth's surface, where $c_{0}$ is practically constant, has to be intended as the basic reference frame for the parameters of light $(\lambda, v)$ while other frames, because of the Doppler effect, would give, for the same source, different values. So, still referring to Figure 1, we intend the frame $\mathrm{O}$ as fixed to the Earth's surface. We also intend:

$T$ : emission time of the considered photon $\mathrm{AB}$.

$\lambda$ : length of the emitting photon if $\mathrm{S}$ is fixed to $\mathrm{O}$ during $T$.

$\lambda_{\mathrm{O}}$ : length of an emitting photon if $\mathrm{S}$ during $T$ is in motion with respect to $\mathrm{O}$.

$T_{t}$ : transit time of one photon ( $T_{\mathrm{O}}$ for $\mathrm{O}, T_{\mathrm{P}}$ for $\mathrm{P}$, while $T_{\mathrm{S}}=T$ ).

On Figure 1, if $\mathrm{S}$ during $T$ is fixed to $\mathrm{O}$, the speed of the front $\mathrm{A}$ (considered as a point-particle), see Equation (3), is $v_{\mathrm{OA}}=u_{\mathrm{O}}$, where $u_{\mathrm{O}}$ only depends on the location of $\mathrm{O}$, thus, at $t=T$, the length $\lambda$ of the photon $\mathrm{AB}$ corresponds to the path covered by A, so we have

$$
v_{\mathrm{OA}} T=u_{\mathrm{O}} T \equiv\left(=v_{\mathrm{SA}} T \text { for } \mathrm{S} \text { fixed to } \mathrm{O} \text { during } T\right) .
$$

If $\mathrm{S}$ has velocity $\mathbf{v}_{\mathrm{OS}}$ with respect to $\mathrm{O}$, the velocity of the front $\mathrm{A}$, referred to $\mathrm{S}$, being $\mathbf{v}_{\mathrm{SA}}=\mathbf{v}_{\mathrm{SO}}+\mathbf{v}_{\mathrm{OA}}$ and since from (3), $\mathbf{v}_{\mathrm{OA}}=\mathbf{u}_{\mathrm{O}}$, becomes

$$
\mathbf{v}_{\mathrm{SA}}=\mathbf{v}_{\mathrm{SO}}+\mathbf{u}_{\mathrm{O}} \Rightarrow v_{\mathrm{SA}}=u_{\mathrm{O}} \pm v_{\mathrm{OS}}
$$

with the sign + if photon and source have opposite direction; then

$$
v_{\mathrm{SA}} T\left(\equiv \lambda_{\mathrm{O}}\right)=u_{\mathrm{O}} T \pm v_{\mathrm{OS}} T \Rightarrow \lambda_{\mathrm{O}}=\lambda(1 \pm \beta) \quad\left[\text { with } \beta=v_{\mathrm{OS}} / u_{\mathrm{O}}\right]
$$

where $\lambda_{\mathrm{O}}$ is the length of the emitted photons when $\mathrm{S}$ is in motion from $\mathrm{O}$. We point out that the variation of the photons length only depends on $\mathbf{v}_{\mathrm{OS}}$.

Now, the transit time $T_{t}$ for the Observer $\mathrm{O}$, can be written

$$
T_{\mathrm{O}}=T \pm v_{\mathrm{OS}} T / u_{\mathrm{O}}=T(1 \pm \beta) \text { [with } \beta=v_{\mathrm{OS}} / u_{\mathrm{O}} \text { ] }
$$

corresponding to the algebraic sum of the time $T\left(=\lambda / u_{\mathrm{O}}\right)$ the front A needs to cover the path $\lambda$, plus the time the tail B (emitted, like every part of the photon, at speed $u_{\mathrm{O}}$ referred to $\mathrm{O}$ ), needs to cover the path $\mathrm{S}_{\mathrm{T}}-\mathrm{O}=v_{\mathrm{OS}} T$, that is $\Delta T=$ $v_{\mathrm{OS}} T / u_{\mathrm{O}}$. (As for the observer $\mathrm{P}$ showed on Figure 1, being $\mathbf{v}_{\mathrm{OP}}=0$, then $T_{\mathrm{P}}=$ $\left.T_{\mathrm{O}}\right)$.

Thus, the speed of the photon $\mathrm{AB}$ referred to $\mathrm{O}$, since $T_{\mathrm{O}}$ increases/decreases together with $\lambda_{O}$, as per Equation (14), becomes 


$$
u_{\mathrm{O}}=\frac{\lambda_{\mathrm{O}}}{T_{\mathrm{O}}}=\frac{\lambda(1 \pm \beta)}{T(1 \pm \beta)}=\frac{\lambda}{T} \equiv c
$$

showing that the speed of a photon referred to an Observer fixed to the source initial location, $(\mathrm{O})$, is invariant despite any motion of the source with respect to O.

The frequency of the light referred to a generic Observer $\mathrm{O}$, on our bases, has to be defined as $v=n / T_{t}$ with $n$ the number of photons, of the same ray, crossing the Observer during their transit time $T_{t}$. Hence, for $T_{t}=1 \mathrm{~s}$, we have $v=n$ meaning that the frequency corresponds to the number of photons, same ray, transiting in $1 \mathrm{~s}$, while, for $n=1$, the frequency becomes

$$
v=1 / T_{t}
$$

We point out that the photons frequency emitted by a source $S$, under the condition $v_{\mathrm{OS}}=0$, has to be equal to the one observed by $\mathrm{O}$; this is also valid if $\mathrm{O}$ and $\mathrm{S}$ belong to different potential, (e.g., the equality of the number of balls falling from the top of a tower with respect to an Observer at the tower base), and therefore the condition $v_{\mathrm{OS}}=0$ always implies $v_{\mathrm{S}}=v_{\mathrm{O}}$ whatever is the distance source-observer.

[We also point out that, according to the Relativity, (due to the claimed constancy of $c$ and because of the Doppler effect), an Observer in relative motion from a source should observe different wavelength as well as different frequency for emitted light; on the contrary, on our results, if the source is fixed to its initial location (the ground on Earth), the length of the emitted photons is invariant, while $c$ and $v$ are varying, as hereafter shown].

Referring now to Figure 2, let $\mathrm{P}$ be an observer (represented at different times, by $\mathrm{P}_{\mathrm{A}}$ and $\mathrm{P}_{\mathrm{B}}$ ) moving with velocity $\mathbf{v}_{\mathrm{OP}}$ from $\mathrm{O}$ along the same direction of the photons while the source $S$ is moving from $O$ with velocity $\mathbf{v}_{O S}$ contrary to them; we assume the photon $A B$ totally emitted at the time the front $A$ reaches $P_{A}$ (for simplicity as $t=0$ ). [In fact, after this emssion, the source may even disappear].

Now, the transit time $T_{\mathrm{P}}$ of the photon $\mathrm{AB}$ is given, see Equation (18), by $T_{\mathrm{O}}$ plus the time $T_{\mathrm{OP}}\left(= \pm v_{\mathrm{OP}} T / c\right)$ due to the motion of $\mathrm{P}$ from $\mathrm{O}$, and since $\mathbf{v}_{\mathrm{SP}}=$ $\mathbf{v}_{\mathrm{SO}}+\mathbf{v}_{\mathrm{OP}}$, we can write

$$
T_{\mathrm{p}}=T \pm \frac{v_{\mathrm{SO}} T}{c} \pm \frac{v_{\mathrm{OP}} T}{c}\left(=T \pm \frac{v_{\mathrm{SP}} T}{c}\right)=T\left(1 \pm \beta \pm \beta_{\mathrm{P}}\right),\left[\text { with } \beta_{\mathrm{p}}= \pm \frac{v_{\mathrm{OP}}}{c}\right]
$$

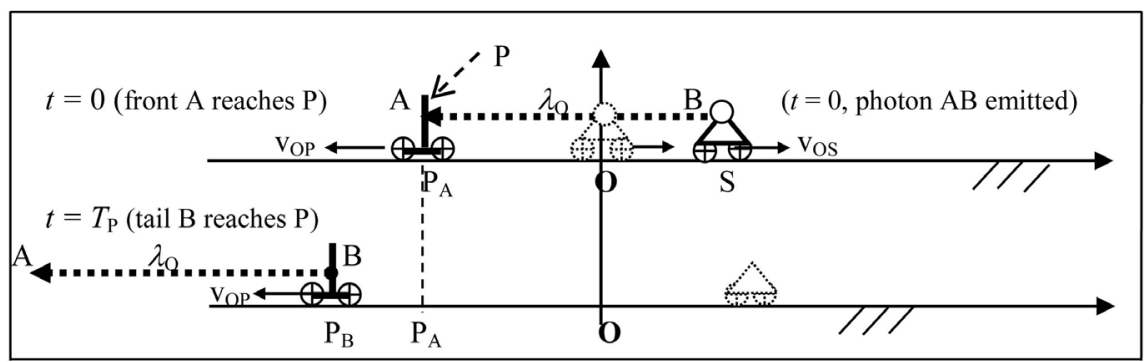

Figure 2. The Observer $\mathrm{P}$ moves from $\mathrm{P}_{\mathrm{A}}$ to $\mathrm{P}_{\mathrm{B}}$ during the transit time $T_{\mathrm{P}}$ of the photon $\mathrm{AB}$ while the source is moving from its initial emission location $\mathrm{O}$. 
corresponding to the interval between the time the front $\mathrm{A}$ reaches $\mathrm{P}_{\mathrm{A}}($ at $t=0$ ) and the time the tail $\mathrm{B}$ leaves $\mathrm{P}_{\mathrm{B}}$; hence

$$
c_{\mathrm{p}}=\lambda_{\mathrm{O}} / T_{\mathrm{p}}=\lambda(1 \pm \beta) / T\left(1 \pm \beta \pm \beta_{\mathrm{p}}\right)=c(1 \pm \beta) /\left(1 \pm \beta \pm \beta_{\mathrm{p}}\right) .
$$

[In short, the motion of $\mathrm{S}$ (from $\mathrm{O}$ ) causes a variation of the photon length, while the relative motion observer-source varies the observer transit time and its related frequency].

On next Figure 3, we analyse the parameters $c, \lambda, v$ of five configurations regarding a source $\mathrm{S}$, the considered frame $\mathrm{O}$ (practically the Earth's surface) and an Observer P.

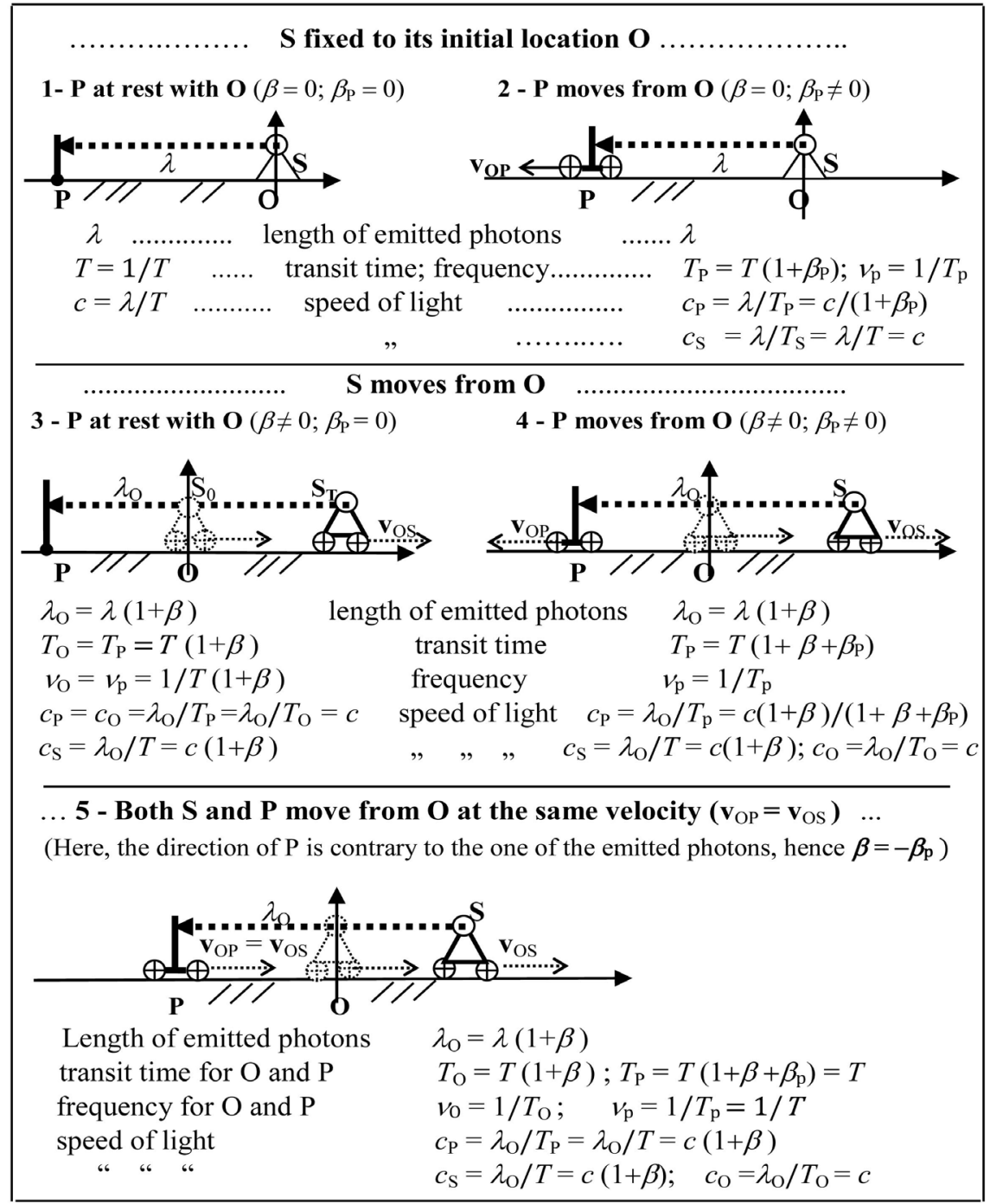

Figure 3. Five configurations regarding a source $S$, a frame $\mathrm{O}$ and an Observer $P$.

where

$\beta=v_{\mathrm{OS}} / c$, relative speed of $\mathrm{S}$ with respect to $\mathrm{O}$,

$\beta_{\mathrm{p}}=v_{\mathrm{OP}} / c$ relative speed of $\mathrm{P}$,

$c=\lambda / T$ basic speed of light, referred to conf. \#1, 
$\lambda_{\mathrm{O}}=\lambda(1 \pm \beta)$ length of emitted photon,

$T_{\mathrm{S}}=T$ photon transit time for $\mathrm{S}$, equal to its emission time,

$T_{\mathrm{O}}=T(1 \pm \beta) ; T_{\mathrm{t}}$ for $\mathrm{O}$; sign + if $\mathrm{S}$ and photon have opposite direction,

$T_{\mathrm{P}}=T\left(1 \pm \beta \pm \beta_{\mathrm{p}}\right) ; T_{\mathrm{t}}$ for $\mathrm{P} ;+\beta_{\mathrm{p}}$ if $\mathrm{P}$ and photon have same direction,

$c_{\mathrm{S}}=\lambda_{\mathrm{O}} / T_{\mathrm{S}}=\lambda_{\mathrm{O}} / T$; speed of light observed by $\mathrm{S}$,

$c_{\mathrm{P}}=\lambda_{\mathrm{O}} / T_{\mathrm{P}}$ speed of light observed by $\mathrm{P}$,

$c_{\mathrm{O}}=\lambda_{\mathrm{O}} / T_{\mathrm{O}}$ speed of light observed by $\mathrm{O}$.

Regarding the measurements of $c$ through the method $c=d / t$ carried out along a double path, the light emitted inside the measurement system (MS), because of the interaction light-matter, has to be absorbed and then re-emitted by the $2^{\text {nd }}$ Observer which, during the re-emission, becomes a new emitting source; therefore the above equations regarding transit time and photon length, are also valid for the re-emitted photons which now have contrary direction, hence $\beta^{\prime}=-\beta$ and also $\lambda^{\prime}=-\lambda_{\mathrm{O}}$ giving to $c$, along a double path, the constant value $c$.

[Light emitted outside the MS: at the time the light is crossing the MS, this becomes the new emitting source].

\subsection{Doppler Effect for the Light, New Equations}

The Equation (17) represents our longitudinal Doppler effect for the light, while the general case of this effect, assuming $v_{\mathrm{OS}}<<$ (hence $\mathrm{OP} \cong \mathrm{PB}$ ), see Figure $4(a)$, can be written as

$$
\lambda_{0}=\lambda \pm v_{\mathrm{OS}} T \cos \alpha=\lambda(1 \pm \beta \cos \alpha) \quad\left(\text { with } \beta=v_{\mathrm{OS}} / c\right)
$$

with $\alpha$ the angle between the direction of $\lambda_{\mathrm{O}}$ and $\mathbf{v}_{\mathrm{OS}}$.

As for the Transverse Doppler effect, see Figure 4(b), in general, we have

$$
\lambda_{0}=\sqrt{\lambda^{2} \pm\left(v_{\mathrm{OS}} T\right)^{2}}=\lambda \sqrt{1 \pm \beta^{2}}
$$

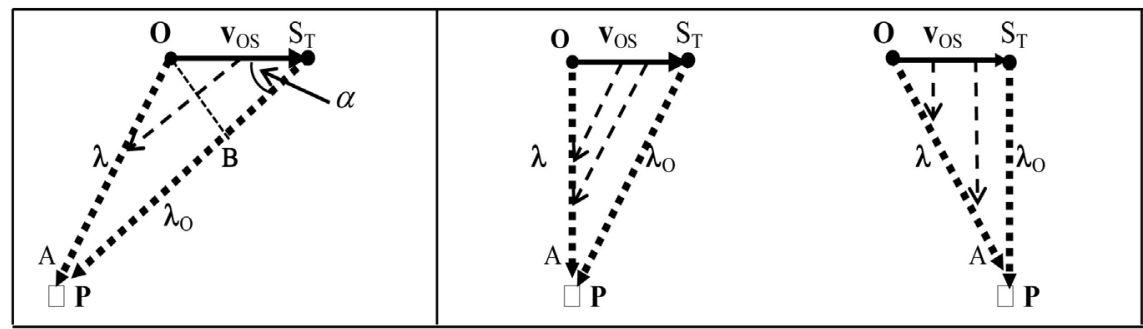

(a)

(b)

Figure 4. Doppler effect (DE). (a) General case; (b) Transverse DE with S receding/approaching an observer P. Dotted arrows inside the triangles represent the photon $\lambda_{\mathrm{O}}$ during its emission.

As for a source circling around an Observer $\mathrm{P}$, the Figure 5 shows that the length of the photon $\lambda$ emitted when its source is fixed to $\mathrm{O}$, becomes $\lambda_{\mathrm{O}}$ when $\mathrm{S}$ is circling with velocity $\mathbf{v}_{\mathrm{OS}}$. 


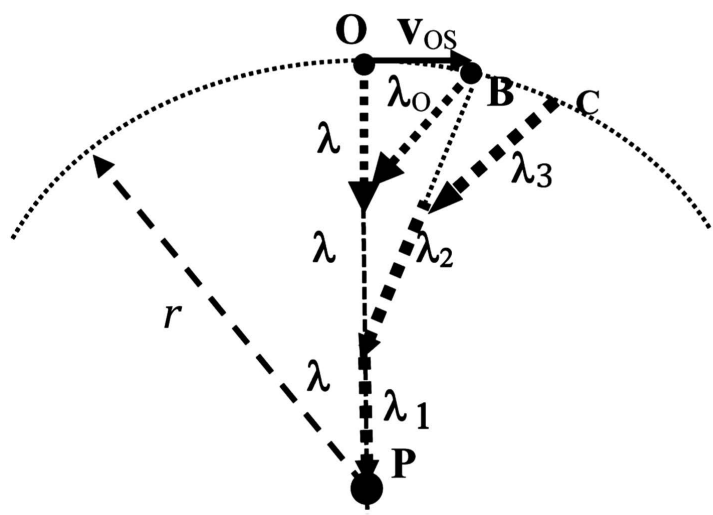

Figure 5. Photons emitted while their source is circling around P.

[The succession of the photons $\lambda$ represents a ray if $\mathrm{S}$ is at rest with $\mathrm{P}$, while the succession $\lambda_{1} \rightarrow \lambda_{3}$ represents a ray if $S$ is circling around $\mathrm{P}$, with $\mathrm{O}, \mathrm{B}, \mathrm{C}$ the emission points of the circling source]. Then, assuming $\lambda_{\mathrm{O}}>\lambda$, we have

$$
\lambda_{\mathrm{O}}=\sqrt{\lambda^{2}+\left(v_{\mathrm{OS}} T\right)^{2}}=\lambda \sqrt{1+\beta^{2}}=\lambda \sqrt{1+\omega^{2} r^{2} / c^{2}}
$$

while the photon transit time, being $T$ the emission time of $\lambda$ becomes

$$
T_{\mathrm{O}}=T \sqrt{1+\beta^{2}}=T \sqrt{1+\omega^{2} r^{2} / c^{2}}
$$

with $r$ the orbit radius, $\omega$ the source angular speed, giving to any photon, speed $c_{\mathrm{O}}=c$.

\subsection{Re-Visitation of the Harvard Tower Experiment (HTE), Time Dilation, Gravitational Redshift}

General Relativity predicts that the gravitational field of the Earth will cause a photon emitted downwards (towards the Earth) to be blueshifted: scope of HTE experiment was to detect this shift of light. A Mossbauer source $S$ was placed on the top of a tower (height $h \cong 22 \mathrm{~m}$ ) emitting photons toward the tower base where an Absorber went out of resonance; the experiment did not clarify if the non-resonance was due to a variation of $\lambda$ or $v$, indeed, because of the claimed constancy of $c$, both of them should have varied their initial values during the path tower-base.

According to our bases, we have different evaluations of this experiment. Let us first consider $\mathrm{S}$ at the base and $\mathrm{A}$ at its top, see Figure 6.

It is a fact that if $S$ and $A$ are at relative rest at the same level (e.g. both on the ground), then $\mathrm{A}$ is in resonance and it is also a fact that other experiments effected at this regard, e.g. Blatt, [7], show that if $S$ moves toward the absorber, not contrarily, the absorber goes out of resonance: indeed, if $S$ is fixed to the ground, the length of emitted photons (as previously shown) is constant, whereas if S moves with respect to its initial emission location (the ground), the photon length varies, thus we may infer that the resonance is physically related to the constancy of the length of photons. 


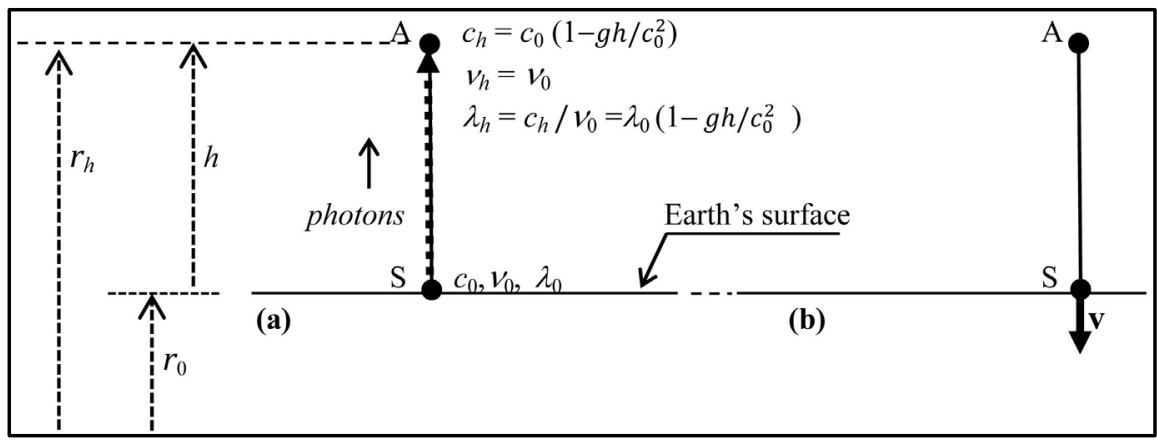

Figure 6. Harvard tower experiment scheme; source at the base, absorber A at the top, our results. (a) S and A at rest, so $v_{h}=v_{0}$; $0, v_{0}, \lambda_{0}$ parameters of photons emitted by $S$ on the ground; $c_{h}, v_{h}, \lambda_{h}$ photons arrived to $A$ where $\lambda_{h}<\lambda_{0}$ thus a blue shift as for A; (b) Direction of the compensating velocity to balance the blue shift via Doppler effect, so to obtain in altitude $c_{0}, v_{0}, \lambda_{0}$.

Now, referring to Figure 6, if $\mathrm{S}$, on the ground, emits photons reaching $\mathrm{A}$ at height $h$, because of the variation of potential, following our relation $c=(-2 U)^{1 / 2}$, it will be $c_{0} \rightarrow c_{h}$; since $\mathrm{S}$ and $\mathrm{A}$ are at reciprocal rest, it turns out that, as shown just after the Equation (20), it is always $v_{h}=v_{0}$ (in spite of any variation of potential between $\mathrm{S}$ and A) yielding $\lambda_{h}=c_{h} / v_{h}$. Therefore, with $r_{0}$ the radius of Earth (hence $r_{h}=r_{0}+h$ ) and $M_{\mathrm{E}}$ the mass of Earth, writing the (11) as $c^{2}=-2 U$, we get $2 c=-2 \mathrm{~d} U / \mathrm{d} c$ hence from $r_{0}$ to $r_{h}$ and since $\Delta c=-\Delta U / c$ with $\Delta U=U_{\mathrm{E} h}-$ $U_{\mathrm{E} 0}=-\left(M_{\mathrm{E}} G / r_{h}\right)+M_{\mathrm{E}} G / r_{0}$, we find

$$
\frac{c_{h}-c_{0}}{c_{0}}=-\frac{\Delta U}{c_{0}^{2}}=-\frac{M_{E} G h}{r_{h} r_{0} c_{0}^{2}} \cong-g h / c_{0}^{2} \Rightarrow c_{h}=c_{0}\left(1-g h / c_{0}^{2}\right)
$$

(where $\Delta U=g h$ ); then for $v_{h}=v_{0}$ we have

$$
\begin{aligned}
& \lambda_{h}=c_{h} / v_{h}=c_{0}\left(1-g h / c_{0}^{2}\right) / v_{0}=\lambda_{0}\left(1-g h / c_{0}^{2}\right) \\
& \Rightarrow\left(\lambda_{h}-\lambda_{0}\right) / \lambda_{0}=-g h / c_{0}^{2}=\frac{c_{h}-c_{0}}{c_{0}}
\end{aligned}
$$

and since $\lambda_{h}<\lambda_{0}$, contrary to Relativity, A observes a blue-shift effect.

Thus, to restore the resonance via Doppler Effect (DE), see Figure 6(b), $S$ has to recede from $\mathrm{A}$ in order that the photon length could increase, see Equation (17), from $\lambda_{h}$ to $\lambda_{0}=\lambda_{h}(1+\beta)$ with $\beta=v_{\mathrm{AS}} / c \equiv v / c$; therefore, equating the relative variation of the photon length due to the $\mathrm{DE}$, that is $\left(\lambda_{h}-\lambda_{0}\right) / \lambda_{0}=-v / c$, to the one due to the altitude, $\Delta \lambda / \lambda_{0}$, as given by (28), we get

$$
v / c=g h / c_{0}^{2} \Rightarrow v=g h / c_{0}=7.5 \times 10^{-7} \mathrm{~m} / \mathrm{s} \quad(\text { for } h=22.5 \mathrm{~m})
$$

i.e. the value of the compensating velocity.

This value is also predicted by GR which, implying a decrease of $v$ for the light moving from the base to the top, predicts an opposite direction of the compensating velocity $\mathrm{v}$ with respect to the one shown on Figure 6(b); at this regard, Pound-Rebka and Pound-Snider, [8] [9] [10], gave no clear indication about the direction of $\mathbf{v}$, since $S$ and $A$, during the experiment, were moved sinusoidally.

Now, see Figure 7, with $A$ on the base, and taking $S$ to the top, the experience 


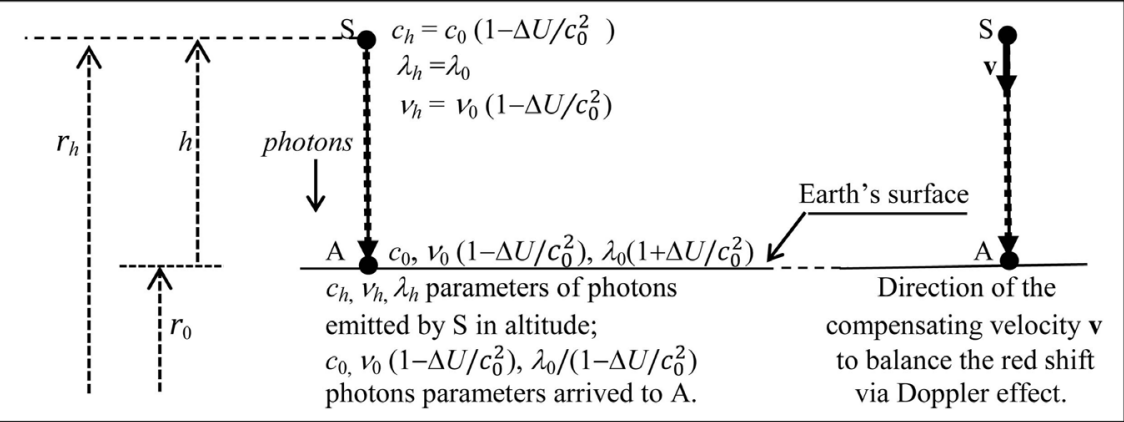

Figure 7. Harvard tower experiment scheme; source on the top, our results.

shows that the absorber goes out of resonance. Indeed, with $S$ on the top, the (27) shows $c_{h}<c_{0}$, but what about the initial parameters of the photons emitted in altitude, $v_{h}$ and $\lambda_{h}$ ?

Well, it is known that atomic clocks give an increase of their counted time in altitude $\left(T_{h}>T_{0}\right.$, hence $v_{h}<v_{0}$ ), therefore taking these clocks from ground to $h$, their frequency $v_{h}$ has to follow the same decrease as $c_{h}$; this implies $\lambda_{h}=\lambda_{0}$ so the frequency of photons emitted in altitude becomes

$$
v_{h}=\frac{c_{h}}{\lambda_{h}}=\frac{c_{0}\left(1-\frac{g h}{c_{0}^{2}}\right)}{\lambda_{0}}=v_{0}\left(1-\frac{g h}{c_{0}^{2}}\right)=v_{0}\left(1-\Delta U / c_{0}^{2}\right) .
$$

Now, with $\mathrm{S}$ emitting photons toward the base, since $\mathrm{S}$ and $\mathrm{A}$ are at rest, their frequency remains constant during their path top-base, meaning that $v_{h-0}=v_{0}\left(1-\Delta U / c_{0}^{2}\right)$, while the length of photons reaching the base, and considering that now $h$ is negative, becomes

$$
\lambda_{h-0}=c_{0} / v_{h-0}=c_{0} / v_{0}\left(1-\Delta U / c_{0}^{2}\right)=\lambda_{0}\left(1+g h / c_{0}^{2}\right)
$$

showing an increase of $\lambda$ from the top to the base. Hence the absorber, on the base, will state, contrary to $G R$, a red-shift, hence, to compensate it via Doppler shift, see Figure 7, S has now to move toward A; on the contrary, according to $G R, A$ and $S$ should recede from each other. We also highlight two points:

- The increase of $\lambda$ for photons moving from $h$ to the ground, gives a reason to the gravitational red shift related to far sources.

- The decrease of $v$ during the path $h$-ground gives a reason to the lower frequency of the light coming from far sources.

Time dilation: Atomic clocks in altitude ( $h$-clocks) are ticking faster than identical clocks on the ground (g-clocks): at height $h$, see (27), we have $\left(c_{h}-c_{0}\right) / c_{0}=-\Delta U / c_{0}^{2}$, while taking a clock to a GPS satellite, see also Figure 7 (a), from (30), one finds

$$
T_{h}=T_{0} /\left(1-\frac{\Delta U}{c_{0}^{2}}\right)=T_{0} /(1-\delta) \Rightarrow T_{h}-\delta T_{h}=T_{0} \quad\left[\text { where } \frac{\Delta U}{c_{0}^{2}} \equiv \delta\right]
$$

with $T_{h}$ the counted time of one photon emitted by a $h$-clock, while $T_{0}$ by a g-clock. Thus the variation of the counted time between the two clocks, for every 
emitted photon, due to the altitude, becomes

$$
\Delta T_{\mathrm{ph}}=T_{h}-T_{0}=T_{h} \delta
$$

and since the frequency of photons is their number emitted in $1 \mathrm{~s}$ along one ray, the term

$$
n_{1 \mathrm{~s}} \equiv v_{0} t_{1 \mathrm{~s}} \quad\left(\text { with } t_{1 \mathrm{~s}}=1 \mathrm{~s}\right)
$$

is the number of photons (atomic transition of Cs 137) constituting one-second on the ground; then, since $v_{h}=v_{0}\left(1-\Delta U / c_{0}^{2}\right)$, see (30), the following relation

$$
\Delta n_{1 \mathrm{~s}}=v_{h} \Delta T_{\mathrm{ph}}=\frac{v_{0}\left(1-\frac{\Delta U}{c_{0}^{2}}\right)\left(T_{0} \frac{\Delta U}{c_{0}^{2}}\right)}{1-\frac{\Delta U}{c_{0}^{2}}}=v_{0} T_{0} \frac{\Delta U}{c_{0}^{2}}=\frac{\Delta U}{c_{0}^{2}}
$$

is the variation of $n_{1 \mathrm{~s}}$ emitted in $1 \mathrm{~s}$ by a $h$-clock; now, see (27), $\Delta U=$ $M_{\mathrm{E}} G h / r_{h} r_{0}$ and since $r_{h} \cong 26,600 \mathrm{~km}, r_{0} \cong 6400 \mathrm{~km}$, with $h \cong 20,200 \mathrm{~km}$, the increase of counted time in one day $\left(\Delta T_{1 d}\right)$ of a $h$-clock, with respect to a g-clock, becomes

$$
\Delta T_{1 \mathrm{~d}}=\Delta n_{1 \mathrm{~s}} \times 86400 \mathrm{~s}=86400 \Delta U / c_{0}^{2}=45.5 \mu \mathrm{s}
$$

hence this effect is a consequence of the variation of $U$; indeed, as shown later on, the values of the $\mathrm{H}$ atom frequencies depend on $c$, thus, on our bases, on $U$.

Now let us find the variation of the counted time, between the two clocks, due to their relative motion; thus, being $v_{\mathrm{S}}=2\left(2 \pi r_{h} / 86,400=3870 \mathrm{~m} / \mathrm{s}\right.$ the orbital speed of GPS satellites, (corresponding to two orbits/day), it turns out that the counted time variation, between a $h$-clock and an Observer $\mathrm{E}$ at the centre of Earth, due to their relative motion, is given by Equation (26) which, in our case, with $\beta_{\mathrm{S}}=v_{\mathrm{S}} / \mathcal{C}\left(\cong 1.3 \times 10^{-5}\right)$ becomes

$$
T_{\mathrm{E}}=T_{h} \sqrt{1+\beta_{\mathrm{S}}^{2}} \cong T_{h}\left(1+\frac{\beta_{\mathrm{s}}^{2}}{2}\right) \quad\left(\text { valid for } \beta_{\mathrm{S}}^{2}<<1\right)
$$

with $T_{E}$ the photon counted time for the Observer E; then, with $V_{0}$ the Earth's rotational speed $\left(\cong 460 \mathrm{~m} / \mathrm{s}\right.$ at the equator), since $\beta_{0}^{2}=\left(v_{0} / c\right)^{2}<<\beta_{\mathrm{S}}^{2}=\left(v_{\mathrm{S}} / c\right)^{2}$ and calling $T_{0} \cong T_{h}\left(1+\beta_{0}^{2}\right)$ the photon counted time of a g-clock with respect to a clock at the Earth's centre E, we can write $T_{0} \cong T_{E}$; thus, the difference between the two transit times $T_{h}$ and $T_{0}\left(\cong T_{E}\right.$ ) due to their relative motion, is

$$
\Delta T_{\mathrm{ph}}^{\prime}=T_{h}-T_{0} \cong T_{h}-T_{\mathrm{E}}=T_{h}-T_{h}\left(1+\frac{\beta_{\mathrm{s}}^{2}}{2}\right)=-T_{h} \beta_{\mathrm{S}}^{2} / 2 .
$$

Then, as above, $\Delta n_{1 \mathrm{~s}}^{\prime} \equiv v_{h} \Delta T_{\mathrm{ph}}^{\prime}=-v_{h} T_{h} \beta_{\mathrm{S}}^{2} / 2=-\beta_{\mathrm{S}}^{2} / 2$ is the variation of the number of photons emitted by a $h$-clock in $1 \mathrm{~s}$; so the variation of the counted time, in one day, is

$$
\Delta T_{1 \mathrm{~d}}^{\prime}=-\left(\frac{\beta_{\mathrm{S}}^{2}}{2}\right) 86400 \mathrm{~s}=-7.2 \mu \mathrm{s}
$$

showing a decrease of the counted time for a g-clock due to their relative mo- 
tion; hence

$$
\Delta T_{\text {totld }}=\Delta T_{1 \mathrm{~d}}+\Delta T_{\mathrm{ld}}^{\prime}=38.3 \mu \mathrm{s} / \text { day }
$$

which is also predicted, (with different reason), by GR. To prevent this effect, before launching, the daily counted time $\left(T_{1 \mathrm{~d}}\right)$ of clocks, has to be decreased by $\cong 38 \mu \mathrm{s}$.

In short, the time dilation is a consequence of the variation of $U$, hence of $c$. indeed, as show on $\$ 2.3$, the admitted/emitted frequency on $\mathrm{H}$ atom (and of course the one of the other elements) varies together with $c$.

\section{Gravitational redshift}

As for the Relativity, the only way to explain high cosmological redshifts is the Doppler effect, (which implies an incredible universe expansion at a speed $v_{\mathrm{u}} \cong$ c), whereas, on our results, disregarding the reciprocal motion between a (far) source and an Observer on Earth, which implies $v=v_{0}$, we get $c / \lambda=c_{0} / \lambda_{0}$, where $v_{0}, c_{0}$ and $\lambda_{0}$ are the values on Earth, hence for $c_{0}>c$ one gets $\lambda_{0}>\lambda$, that is a red shift observed on Earth; therefore, the shifts here observed can be expressed as

$$
z \equiv \frac{\Delta \lambda}{\lambda}=\frac{\Delta c}{c}=\frac{c_{0}-c}{c}=\frac{c_{0}}{c}-1=\sqrt{U_{0} / U}-1
$$

with $U_{0}$ the potential on Earth, $U$ the one on the source. Thus, apart from Doppler effects, $z$ turns out to be the variation of $c$ (as well as $\lambda$ ) during the path of light toward a different potential. In particular, with $s$ the distance Earth-source, for $s<\cong 45 \mathrm{Mpc}$, [11], (roughly corresponding to $-0.01<z<$ +0.01 ) if $U$ (potential on the source) is, in absolute value, higher than the potential on Earth $U_{0}$, the (41) gives, on Earth, $z<0$ (blue shift), and vice versa for $|U|$ $<\left|U_{0}\right|$; thus, for $s<\cong 45 \mathrm{Mpc}$, these red/blue shifts indicate that the potential, may increase or decrease with respect to $\left|U_{0}\right|$. In the range $\cong 0.01<z<\cong 0.20$, (where $z$ follows the Hubble's law), the (41), written as

$$
U=U_{0} /(1+z)^{2} \cong U_{0} /(1+2 z) \cong U_{0}(1-2 z) \quad(\text { valid for } z<<1)
$$

shows that, for $z<<1, U$ depends linearly on $z$, as Hubble's law; then, for $s \rightarrow \infty$, $U \rightarrow 0$, hence $z \rightarrow \infty$, see Table 1 .

[For $s>\cong 45 \mathrm{Mpc}, z$ is always positive, hence we may infer that our galaxy is practically near/close to the middle of the masses of universe (where $|U|$ has the

\begin{tabular}{|c|c|c|c|c|c|}
\hline blue/redshift & $\mathrm{z}$ & $s(\mathrm{Mpc})$ & $U / U_{0}=1 /(z+1)^{2}$ & $\begin{array}{c}U / U_{0} \cong 1-2 z \\
\text { valid for } \mathrm{z}<<1\end{array}$ & $c / c_{0} \cong 1 /(z+1)$ \\
\hline blue/red shift & $-0.01 \rightarrow 0.01$ & $<\cong 45$ & $0.98-1.02$ & $0.98-1.02$ & $0.99-1.01$ \\
\hline red shift & $\cong 0.01$ & $\cong 45$ & 0.98 & 0.98 & 0.99 \\
\hline red shift & 0.20 & 900 & 0.69 & 0.60 & 0.83 \\
\hline red shift & 1 & & 0.25 & & 0.50 \\
\hline red shift & $\rightarrow \infty$ & $\rightarrow \infty$ & $\rightarrow 0$ & & $\rightarrow 0$ \\
\hline
\end{tabular}
max value)].

Table 1. Calculated values of $U$ and $c$ related to the observed shifts on earth. 


\section{Interaction Light-Matter}

\subsection{Electron Structure and Photon-Electron Impact Point}

On our basis, (light composed of our photons), the interaction light-matter requires that to move a circling electron toward outer orbits, the impact photon-electron has to occur, see Figure 8(a), in a radial way, (giving origin to the radial velocity $\mathbf{w}$ ), otherwise, other impacts could cause the electron fall into the nucleus, due, for instance, to an impact where the velocity of photons and electron have contrary direction.

To be radial, the impact must occur in a specific point, fixed to the electron surface, we call it Impact Point, which, during the electron, orbit, has to face its nucleus, (up to its removal), giving to the electron one rotation every orbit; the impacting photons have to approach the nucleus, as shown on Figure $8(\mathrm{~b})$, perpendicularly to the electron orbit plane, providing, to the electron, a radial velocity $\mathbf{w}$.

Moreover, we can infer that the charge of the electron, has to correspond to the Impact Point $\left(\mathrm{I}_{\mathrm{p}}\right)$, so we may infer that each photon front is provided with a positive charge, while its tail with an equal negative one. In fact, the photon may be represented as an electric bipole.

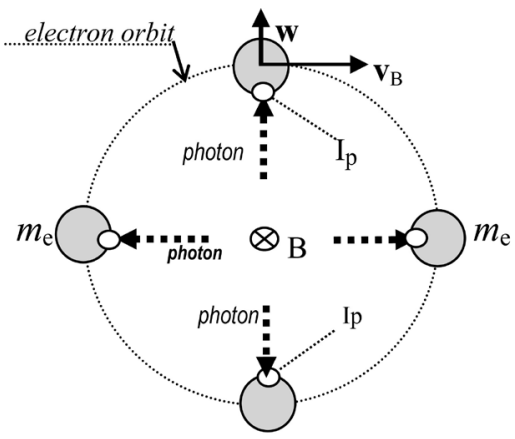

(a)

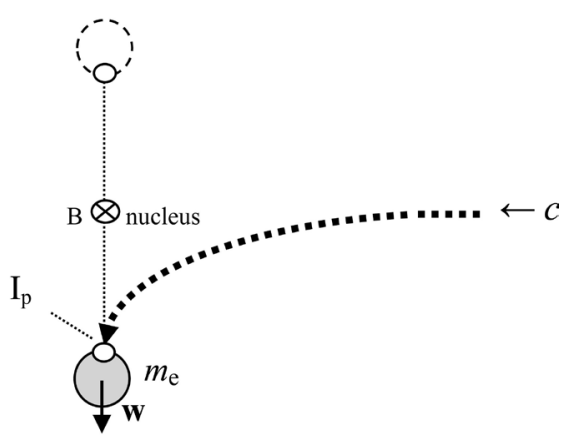

(b)

Figure 8. Photon-electron Impact point $\left(\mathrm{I}_{\mathrm{p}}\right)$ and electron radial velocity w. (a) Electron orbit front view; (b) Side view.

\subsection{Physical Characteristics of Photons}

The total energy of a mass $m$ is $E=m c^{2}$ also proved [12] by the evidence of nuclear reactions like $n+p d+\gamma$, hence this energy has to be valid for the (massive) light too, thus writing their energy as $E=\frac{1}{2} m c^{2}+\frac{1}{2} m c^{2}$ we have to infer that photons are provided, out of their kinetic energy $K_{c}=\frac{1}{2} m c^{2}$ with an internal energy $K_{i}$ equal to $K_{c}$ and we point out that, toward the infinity, (where $U_{\infty}$ $\rightarrow 0$ hence $c_{\infty} \rightarrow 0$ ), both $K_{\mathrm{c}}$ and $K_{\mathrm{i}}$ tend to 0 ; then, since for the light $E=h v$, the following relation

$$
E=K_{c}+K_{i}=m c^{2}=h v
$$

has to represent the energy of one ray of light, (where photons are flowing), and 
where

$$
m=h v / c^{2} \equiv \gamma v
$$

is the mass of light, having frequency $v$, passing along one ray in $1 \mathrm{~s}$, while the constant

$$
\gamma=h / c^{2}=m / v=m T=7.372495 \times 10^{-51} \mathrm{~kg} \mathrm{~s}
$$

represents the mass of one photon crossing an observer during the transit time $T$, while the Planck's constant

$$
h=\gamma c^{2}
$$

turns out to be the energy of one photon (mass of light of one ray passing during T).

Now, since $m$ is the mass of light passing along one ray in $1 \mathrm{~s}$, the term $n_{\mathrm{r}} m c^{2}$, with $n_{\mathrm{r}}$ the number of rays emitted by a source $\mathrm{S}$, becomes the energy emitted by $\mathrm{S}$ in $1 \mathrm{~s}$; this unitary (unit of time) energy shall be equal to the supplied power $P$ during $1 \mathrm{~s}$, yielding

$$
n_{r} m c^{2}=P
$$

therefore

$$
m_{\mathrm{tot}}=n_{r} m=P / c^{2}
$$

is the total mass lost per second by a source of light; e.g. for a $1 \mathrm{~W}$ lamp, we get

$$
m_{\text {tot }}=P / c^{2} \cong 1.1 \times 10^{-17} \mathrm{~kg} \mathrm{~s}^{-1}
$$

while the number $n_{\mathrm{r}}$ of rays is

$$
n_{r}\left(=m_{\mathrm{tot}} / \gamma v\right)=P / c^{2} \gamma v=P / h v
$$

in our case, $n_{r} \cong 3 \times 10^{18}$ rays, and we point out that, as for a given power $P$, the higher is the frequency, the lower is the number of rays, as shown by (50) written as $n_{r} v=P / h$. Then, the number of photons $n_{\gamma}$ emitted in $1 \mathrm{~s}$ becomes

$$
n_{\gamma}=\left(n_{r} v\right)=P v / h v=P / h
$$

which, for $P=1 \mathrm{~W}$, gives $n_{\gamma}=h^{-1}\left(=1.5 \times 10^{33}\right.$ photons/s), thus the inverse of Planck's constant turns out to be the number of photons emitted in 1s by a source of unitary power, and this great number of photons allows the light to be treated as a wave.

Now, during inelastic impacts photons-electron, (like on absorption or photoelectric effetcs), both kinetic and internal energy of the light are involved, so the momentum transferred to the electron is

$$
\mathbf{p}=2 m \mathbf{c}=2 \gamma \nu \mathbf{c}=2 \gamma \mathbf{c} / T
$$

whose validity is confirmed, see Table 2 of chapter 2.4, by the equality $w_{137}=v_{137}$ (with $v_{137}$ the electron orbital speed along the $\mathrm{H}$ atom orbit \# 137 and where $W_{137}$ is the value of the electron radial speed along such orbit, as a condition for the $\mathrm{H}$ atom ionization. 
Regarding elastic impacts, the momentum transferred to the electron, is

$$
\mathbf{p}^{\prime}=m^{\prime} \mathbf{c}=\gamma v^{\prime} \mathbf{c}=\gamma \mathbf{c} / T^{\prime}
$$

either for incident or for reflectedphotons, with $T^{\prime}$ the total impact time for this interaction; through the (53), and also through our Doppler effect equation, see (17), we got the Compton equation (which cannot be obtained by the Relativity via their Doppler effect equations).

\subsection{H Atom Parameters and Meaning of Quantum Numbers}

Figure 9(a) represents, on our bases, the $\mathrm{H}$ atom basic configuration; on the intermediate conf. (b) the electron is represented circling along the same orbit as its charge, while on conf. (c) the electron reduced mass

$m_{r}=m_{\mathrm{e}} /\left(1+m_{\mathrm{e}} / m_{\mathrm{P}}\right)=m_{\mathrm{e}} /\left(1+\varepsilon_{m}\right)$, with $\mathcal{E}_{m}=m_{\mathrm{e}} / m_{\mathrm{p}}$, circling around the proton mass $m_{\mathrm{P}}$ fixed as origin, is represented either along the effective orbit $r_{0}^{\prime}$ or along $r_{0}$; referring to conf. (a) we define:

$m_{\mathrm{e}}$ : electron mass;

$m_{\mathrm{p}}$ : proton mass;

$v_{\mathrm{e}}=\left|\mathbf{v}_{\mathrm{e}}\right|$ electron ground-state (orbital) speed,

$v_{\mathrm{p}}=\left|\mathbf{v}_{\mathrm{P}}\right|$ proton ground-state speed,

$r_{\mathrm{B}}$ the ground-state orbit of the electron charge,

$r_{\mathrm{e}}$ the electron radius,

$\varepsilon_{r} \equiv r_{\mathrm{e}} / r_{\mathrm{B}}$

$r_{\mathrm{B}}^{\prime}=\left(r_{\mathrm{B}}+r_{\mathrm{e}}\right)=r_{\mathrm{B}}\left(1+\varepsilon_{r}\right)$, ground-state orbit of the electron barycentre,

$r_{\mathrm{p}}$ the proton ground-state orbit.

Now, the constancy of momentum referred to conf. Figure 9(a) gives

$$
m_{\mathrm{e}} v_{\mathrm{e}}=m_{\mathrm{p}} v_{\mathrm{p}} \Rightarrow v_{\mathrm{p}}=m_{\mathrm{e}} v_{\mathrm{e}} / m_{\mathrm{p}} \equiv \varepsilon_{m} v_{\mathrm{e}}
$$

then, equating on (a) the frequency of $m_{\mathrm{e}}$ and $m_{\mathrm{p}}$, we get

$$
v_{\mathrm{e}} / r_{\mathrm{B}}^{\prime}=v_{\mathrm{p}} / r_{\mathrm{p}} \Rightarrow r_{\mathrm{p}}\left(v_{\mathrm{p}} / v_{\mathrm{e}}\right) r_{\mathrm{B}}^{\prime}=\varepsilon_{m} r_{\mathrm{B}}^{\prime}=\varepsilon_{m} r_{\mathrm{B}}\left(1+\varepsilon_{r}\right)
$$

The conf. (a), moving $m_{\mathrm{e}}$ (without its charge) from the orbit $r_{\mathrm{B}}^{\prime}$ to $r_{\mathrm{B}}$ (the electric forces acting on $m_{\mathrm{e}}$ are not involved), turns to conf. (b) where the equality between the frequency of $m_{\mathrm{e}}$ and the one of its charge, gives

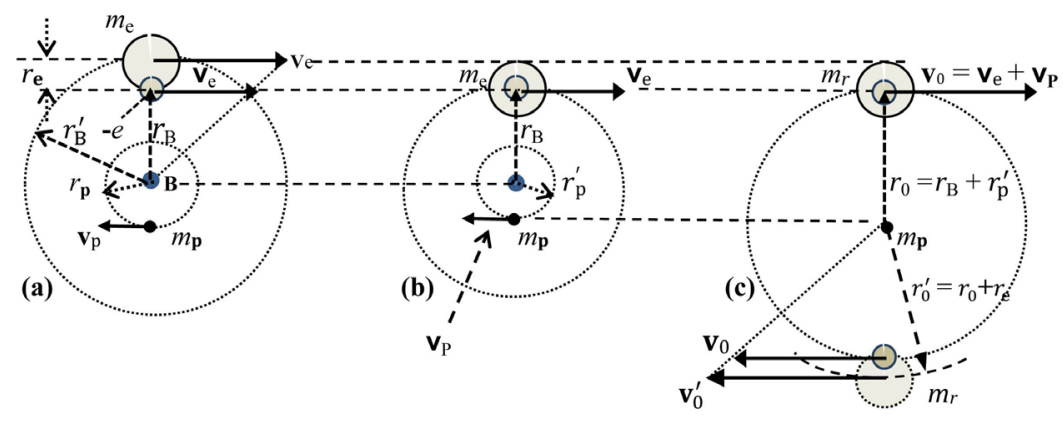

Figure 9. H atom equivalent configurations, on our bases. (a) Observed from the electron-proton common centre of gravity B; (b) Ditto, with the electron barycentre now coincident with its proper charge; (c) Observed from the proton fixed as origin, orbited by the electron reduced mass $m_{r}$ represented either circling along $r_{0}$ or its effective orbit $r_{0}^{\prime}$. 


$$
v_{\mathrm{e}} / r_{\mathrm{B}}^{\prime}=v_{\mathrm{e}} / r_{\mathrm{B}} \Rightarrow v_{\mathrm{e}}=v_{\mathrm{e}} /\left(1+\varepsilon_{r}\right)
$$

then the constancy of momentum referred to conf. (b), $m_{\mathrm{e}} v_{\mathrm{e}}=m_{\mathrm{p}} v_{\mathrm{p}}$, yields

$$
v_{\mathrm{p}}=m_{\mathrm{e}} v_{\mathrm{e}} / m_{\mathrm{p}}=\varepsilon_{m} v_{\mathrm{e}} \Rightarrow v_{\mathrm{p}} / \nu_{\mathrm{e}}=\varepsilon_{m}
$$

then, equating on (b) the frequency of $m_{\mathrm{e}}$ and $m_{\mathrm{p}}$, we get

$$
v_{\mathrm{e}} / r_{\mathrm{B}}=v_{\mathrm{p}} / r_{\mathrm{p}}^{\prime} \Rightarrow r_{\mathrm{p}}^{\prime}=\left(v_{\mathrm{p}} / v_{\mathrm{e}}\right) r_{\mathrm{B}}=\varepsilon_{m} r_{\mathrm{B}}
$$

and finally, see conf. (c), we find

$$
r_{0}=r_{\mathrm{B}}+r_{\mathrm{P}}^{\prime}=r_{\mathrm{B}}+r_{\mathrm{B}} \varepsilon_{m}=r_{\mathrm{B}}\left(1+\varepsilon_{m}\right)=r_{\mathrm{B}}^{\prime}\left(1+\varepsilon_{m}\right) /\left(1+\varepsilon_{r}\right)
$$

where $m_{r}$ is circling with speed $v_{0}=v_{\mathrm{e}}+v_{\mathrm{p}}=v_{\mathrm{e}}\left(1+\varepsilon_{m}\right)$; then

$$
r_{0}^{\prime}=r_{0}+r_{\mathrm{e}}=r_{0}+\varepsilon_{r} r_{\mathrm{B}}=r_{0}\left[1+\varepsilon_{r} /\left(1+\varepsilon_{m}\right)\right]=r_{0}\left(1+\varepsilon_{m}+\varepsilon_{r}\right) /\left(1+\varepsilon_{m}\right)
$$

Now, it is known that, on $\mathrm{H}$ atom, the admitted frequencies have to satisfy the relation

$$
v_{n}=v_{0} / n^{2} \quad(n=1,2,3, \cdots)
$$

with $v_{n}$ the photons admitted frequency along the $n$-th circular orbit and since, as claimed on Section 1.4, $v(=n / t)$ is the number of photons (of the same ray) crossing an observer during the time $t$, the integer $n$ represents the number of photons absorbed by the electron during the photon-electron impact time $t$ and this number, for all the $H$ atom circular orbits, is an integer starting with 1 along the electron ground-state orbit.

The massiveness of light implies a finite impact time during which the electron is circling, and supposing, for now, that, for $n=1$, the photon frequency $v_{0}$ should be equal to the electron frequency along the ground-state orbit, that is $v_{\mathrm{e} 0}$ $=v_{0} / 2 \pi r_{0}$, where $r_{0}$, see conf. (c) is the electron ground-state orbit, with $v_{0}$ its orbital speed, we could write

$$
v_{0} / v_{\mathrm{e} 0}=1 \quad \text { (first assumption); }
$$

to verify this ratio or to find the correct value, we may start from the experimental value of the ${ }^{1} \mathrm{H}$ ground-state ionization energy $W_{0}$ (electron extraction work) corresponding to

$$
W_{0}=-U_{r 0} / 2=e^{2} / 8 \pi \varepsilon_{0} r_{0}=1 / 2 m_{r} v_{0}^{2} \cong 13.8954 \mathrm{eV}
$$

with $U_{r 0}=-e^{2} / 4 \pi \varepsilon_{0} r_{0}$ the potential of the dipole electron-proton, yielding

$$
r_{0}=e^{2} / W_{0} 8 \pi \varepsilon_{0} \cong 5.29461 \times 10^{-11} \mathrm{~m}
$$

(rough value of the Bohr radius), thus the orbital speed of the electron along $r_{0}$ becomes

$$
v_{0}=\sqrt{e^{2} / 4 \pi \varepsilon_{0} r_{0} m_{r}} \cong 2.18770 \times 10^{6} \mathrm{~m} / \mathrm{s}
$$

Then, if $W_{0}$ is supplied by one ray of light (with energy $E=h v_{0}$ ) it must be

$$
1 / 2 m_{r} v_{0}^{2}=h v_{0} \Rightarrow v_{0}=m_{r} v_{0}^{2} / 2 h \cong 3.28808 \times 10^{15} \mathrm{~s}^{-1}
$$

with $v_{0}$ the admitted frequency along $r_{0}$; hence, the ratio $v_{0} / v_{\mathrm{e} 0}$ becomes 


$$
v_{0} / v_{\mathrm{e} 0}=\left(T_{\mathrm{e} 0} / T_{0}\right)=v_{0} 2 \pi r_{0} / v_{0}=0.499998
$$

meaning that during one electron orbit it should transit half of a photon, so we may infer that along $r_{0}$ it should be

$$
2 T_{\mathrm{e} 0} / T_{0}=2 v_{0} / v_{\mathrm{e} 0}=1 \text { exact }
$$

meaning that, along $r_{0}$ the impact time $T_{0}$ lasts for two electron circular orbits.

Now, see conf. (c) equating along $r_{0}$ the electron centrifugal force to the Coulomb force we have

$$
m_{r} v_{0}^{2} / r_{0}=\frac{e^{2}}{4 \pi \varepsilon_{0} r_{0}^{2}} \Rightarrow m_{r} v_{0}^{2}=e^{2} / 4 \pi \varepsilon_{0} r_{0}=-U_{r 0}
$$

which, for a circular orbit, and since, see (66), $m_{r} v^{2}=2 h v$, gives

$$
v_{n}=e^{2} / 4 \pi \varepsilon_{0} r_{n} 2 h
$$

then

$$
r_{n}=e^{2} / 8 \pi \varepsilon_{0} h v_{n}=e^{2} n^{2} / 8 \pi \varepsilon_{0} h v_{0}=r_{0} n^{2}
$$

while as for the orbital speed of the electron $m_{r}$ along any circular orbit $r_{n}$ the (65) gives

$$
v_{n}^{2}=e^{2} / 4 \pi \varepsilon_{0} r_{n} m_{r}=e^{2} / 4 \pi \varepsilon_{0} r_{0} n^{2} m_{r}=v_{0}^{2} / n^{2} \Rightarrow v_{n}=v_{0} / n
$$

and finally the electron frequency along a circular orbit becomes

$$
v_{\mathrm{e} n}=v_{n} / 2 \pi r_{n}=v_{0} / n 2 \pi r_{0} n^{2}=v_{\mathrm{e} 0} / n^{3} .
$$

Now, along $r_{0}$, the (70) yields

$$
v_{0}=e^{2} / 4 \pi \varepsilon_{0} r_{0} 2 h=e^{2} c / 2 \varepsilon_{0} h c 4 \pi r_{0}=\alpha c / 4 \pi r_{0}
$$

with $\alpha=e^{2} / 2 \varepsilon_{0} h c$ the fine structure constant and given $2 v_{0} / v_{\mathrm{e} 0}=1$ (exact) we have

$$
\delta_{1} \equiv 2 v_{0} / v_{\mathrm{e} 0}=\left(2 \alpha c / 4 \pi r_{0}\right) /\left(v_{0} / 2 \pi r_{0}\right)=a c / v_{0}=1
$$

yielding

$$
v_{0}=\alpha c=e^{2} / 2 h \varepsilon_{0}=2187691.2 \mathrm{~m} / \mathrm{s}
$$

and then the (66) becomes

$$
v_{0}=m_{r} v_{0}^{2} / 2 h=m_{r} \alpha^{2} c^{2} / 2 h=R_{\mathrm{H}} c
$$

where $R_{\mathrm{H}}=m_{r} \alpha^{2} c / 2 h=1 / \lambda_{0}$ is the Rydberg constant.

Now, from (61) and (73), because of the ratio $\delta_{1} \equiv 2 v_{0} / v_{\mathrm{e} 0}=1$, we also get

$$
\delta_{n} \equiv 2 v_{n} / v_{\mathrm{e} n}=\left(2 v_{0} / n^{2}\right) /\left(v_{\mathrm{e} 0} / n^{3}\right)=n(\text { with } n=1,2,3, \cdots)
$$

which written as $n T_{n}=2 T_{\text {en }}$ shows that the impact time $n T_{n}$ of $n$ photons (with frequency $v_{n}$ ) equals the time the electron needs for two orbits along any circular orbit $r_{n}$.

Now the correct value of $r_{0}$, from (71), becomes

$$
r_{0}=\frac{e^{2}}{8 \pi \varepsilon_{0} h v_{0}}=\frac{e^{2}}{8 \pi \varepsilon_{0} h R_{\mathrm{H}} c}=\frac{\alpha}{4 \pi R_{\mathrm{H}}}=5.294654 \times 10^{-11} \mathrm{~m}
$$


which should represent the electron ground state orbit if the electron should circle, together with its charge, around $r_{0}$ : indeed the ground-state effective orbit of the electron barycentre observed from $m_{\mathrm{p}}$ is $r_{0}^{\prime}$. Then, writing the (59) as $r_{\mathrm{B}}=r_{0} /\left(1+\varepsilon_{m}\right)$ we get

$$
r_{\mathrm{B}}=\frac{r_{0}}{1+\frac{m_{\mathrm{e}}}{m_{\mathrm{P}}}}=\alpha / 4 \pi R_{\mathrm{H}}\left(1+\varepsilon_{m}\right)=\frac{\alpha}{4 \pi R_{\infty}}=5.291772 \times 10^{-11} \mathrm{~m}
$$

corresponding to the Bohr radius which, on our results, is the orbit of the electron charge referred to $\mathrm{B}$ (centre of mass of the system electron-proton)see Figure 9 (a).

Now, still referring to conf. (c), the effective electron orbit (i.e. its barycenter) is $r_{0}^{\prime}$ where its effective speed is

$$
\begin{aligned}
v_{0}^{\prime} & =\frac{v_{0} r_{0}^{\prime}}{r_{0}}=\frac{v_{0}\left(r_{0}+r_{\mathrm{e}}\right)}{r_{\mathrm{B}}\left(1+\varepsilon_{m}\right)}=v_{0}\left[r_{\mathrm{B}}\left(1+\varepsilon_{m}\right)+\varepsilon_{r} r_{\mathrm{B}}\right] / r_{\mathrm{B}}\left(1+\varepsilon_{m}\right) \\
& =\frac{v_{0}\left(1+\varepsilon_{m}+\varepsilon_{r}\right)}{1+\varepsilon_{m}}=\alpha c\left(1+\frac{\varepsilon_{r}}{1+\varepsilon_{m}}\right)
\end{aligned}
$$

and since the distance between the electron charge and the proton charge is $r_{0}$ (either considering the electron circling along $r_{0}^{\prime}$ or $r_{0}$ ), it turns out that along $r_{0}^{\prime}$ the photon admitted frequency is $v_{0}^{\prime}=v_{0}$, and therefore we get

$$
\delta_{1}^{\prime}=\frac{2 v_{0}}{v_{\mathrm{e} 0}^{\prime}}=2 v_{0} /\left(v_{0}^{\prime} / 2 \pi r_{0}^{\prime}\right)=2 v_{0} / \frac{v_{0} r_{0}^{\prime}}{r_{0}} / 2 \pi r_{0}^{\prime}=2 v_{0} 2 \pi r_{0} / v_{0}=1
$$

meaning one photon every two electron ground-state orbit, and since $r_{0}^{\prime}=\left(r_{0}+r_{\mathrm{e}}\right)$ is also a circular orbit, the effective electron orbit $r_{n}^{\prime}$ has following parameters:

$$
r_{n}^{\prime}=r_{0}^{\prime} n^{2} ; v_{n}^{\prime}=v_{0}^{\prime} / n ; v_{\mathrm{e} n}^{\prime}=v_{\mathrm{e} 0}^{\prime} / n^{3} ; v_{n}^{\prime}=v_{n}
$$

thus for each circular orbit we get

$$
\delta_{n}^{\prime}=\frac{2 v_{n}}{v_{\mathrm{e} n}^{\prime}}=\left(2 v_{0} / n^{2}\right) /\left(v_{\mathrm{e} 0}^{\prime} / n^{3}\right)=2 n v_{0} / v_{\mathrm{e} 0}^{\prime}=n
$$

the same as along $r_{n}$.

\subsection{Electron Radial Speed, Ionization Condition, Electron Radius}

To apply properly the Conservation of momentum (CoM) to the impact photon-electron, the atom nucleus has to be considered fixed as origin in the common centre of gravity nucleus-electrons. On $\mathrm{H}$ atom, referring to Figure 9 (c), an inelastic impact photon-electron, since photon and $\mathbf{w}$, see Figure 8, have same direction, the (52), gives

$$
w=2 m c / m_{r}=2 \gamma v c / m_{r}=2 \gamma v c^{2} / \mathrm{cm}_{r}=2 \mathrm{hv} / \mathrm{cm}_{r}
$$

where the frequency $v$, see (70), depends on the effective distance $r$ between the proton and the electron charge, while the term $w$, see also Figure 10, is the electron radial speed originated by the impact of one photon during the impact time $T(=1 / v)$; for $n$ photons of the same ray the (85) gives 


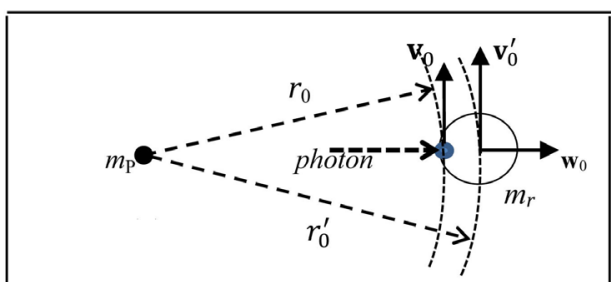

(a)

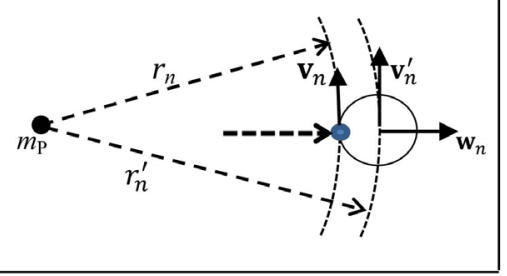

(b)

Figure 10. $\mathrm{H}$ atom: orbital and radial electron speed, see also Figure 9(c). (a) Along $r_{0}$ we have: $v_{0}=\alpha c ; w_{0}=\alpha^{2} c$; then, along $r_{0}^{\prime}$ we will find $v_{0}^{\prime}=c / 137$; (b) Along the ionization orbit: $w_{n^{2}}=v_{n}^{\prime}=c / 137^{2}$.

$$
w_{n}=n w=2 n h v / \mathrm{cm}_{r}
$$

while the generic condition

$$
w=v \quad \text { (ionization condition) }
$$

where $v$ is the electron speed along the ionization orbit, represents the sufficient condition for an electron to escape from its atom, (with zero final velocity of the electron, like on Absorption effect and, of course, for $\mathrm{H}$ atom).

Now, writing the (66) as $v_{0}=m_{r} v_{0}^{2} / 2 h$, the (85) leads to

$$
w_{0}=\frac{2 h m_{r} v_{0}^{2}}{2 h c m_{r}}=v_{0}^{2} / c=\alpha^{2} c=15964.35 \mathrm{~m} / \mathrm{s} \cong c / 137^{2}
$$

thus the ionization condition along $r_{0}$, for one photon only, becomes

$$
\frac{w_{0}}{v_{0}}=\frac{v_{0}}{c}=\alpha \cong 1 / 137 \ll 1
$$

hence the ionization, requiring $w / v=1$, could happen for $n=137$, but the number of admitted photons, along $r_{0}$, is $n=1$. Now, along the progressive orbit $\# n$, the admitted photons frequency is $v_{n}=v_{0} / n^{2}$, and since $n$ is the number of admitted photons along each electron circular d-orbit, we may infer that each progressive orbit is composed of $n$ electron d-orbits, yielding $n^{2}$ admitted photons along each of these orbits.

Referring now to the electron effective ground-state orbit $r_{0}^{\prime}$, regarding the orbit $r_{n}^{\prime} \equiv r_{0}^{\prime} n^{2}$, where $v_{n}^{\prime}=v^{\prime} / n$, the (86), for $n^{2}$ photons, gives

$$
w_{n^{2}}\left(=n w_{\# n}\right)=\frac{n^{2} 2 h v_{n}^{\prime}}{c m_{r}}=\frac{n^{2} 2 h v_{0}^{\prime}}{c m_{r} n^{2}}=\frac{2 h v_{0}^{\prime}}{c m_{r}}
$$

constant along every circular orbit, and where $v_{0}^{\prime}$ from (66) becomes

$$
v_{0}^{\prime}=m_{r} v_{0}^{\prime 2} / 2 h
$$

which represents the photon virtual frequency, intending the admitted frequency if the electron charge would be coincident with the electron barycenter along its effective orbit $r_{0}^{\prime}$; indeed, the photons admitted frequency is related to the effective distance $r_{0}$, where the effective photon frequency is therefore $v_{0}$. In fact, we introduce $v_{0}^{\prime}$ to determine the value of $v_{0}^{\prime}$ (ground-state electron effective orbital speed). 
Therefore the ionization condition along $r_{n}^{\prime}$ becomes

$$
w_{n^{2}}=v_{n}^{\prime}=v_{0}^{\prime} / n
$$

yielding

$$
2 h v_{0}^{\prime} / c m_{r}=v_{0}^{\prime} / n \Rightarrow n=v_{0}^{\prime} c m_{r} / 2 h v_{0}^{\prime}
$$

then

$$
n=v_{0}^{\prime} c m_{r} 2 h / 2 h m_{r} v_{0}^{\prime 2} \Rightarrow n=\frac{c}{v_{0}^{\prime}} \cong \frac{c}{v_{0}}=\frac{c}{\alpha c} \cong 137
$$

but $n$ has to be in integer, so we can infer $n=c / v_{0}^{\prime}=137$ and therefore we get

$$
v_{0}^{\prime}=c / 137
$$

representing the electron speed along its effective ground-state orbit, while

$$
v_{137}^{\prime}=v_{0}^{\prime} / 137=c / 137^{2}
$$

represents the electron speed along its ionization orbit, see also Table 2.

Then, since $v_{0}^{\prime}=\alpha c\left(1+\frac{\varepsilon_{r}}{1+\varepsilon_{m}}\right)$ we obtain

$$
\frac{c}{137}=\frac{\alpha c\left(1+\varepsilon_{m}+\varepsilon_{r}\right)}{1+\varepsilon_{m}}
$$

then

$$
1+\varepsilon_{m}+\varepsilon_{r}=\frac{\left(1+\varepsilon_{m}\right)}{137 \alpha} \Rightarrow \varepsilon_{r}=\left(1+\varepsilon_{m}\right)\left(\frac{1}{137 \alpha}-1\right)=0.0002629141
$$

and since, for definition, $r_{\mathrm{e}}=\varepsilon_{r} r_{\mathrm{B}}$, we get

$$
r_{\mathrm{e}}=\varepsilon_{r} r_{\mathrm{B}}=\frac{\varepsilon_{r} \alpha}{4 \pi R_{\infty}}=\left(1+\varepsilon_{m}\right)\left(\frac{1}{137 \alpha}-1\right) \alpha / 4 \pi R_{\infty}=\left(\frac{1}{137 \alpha}-1\right) \alpha / 4 \pi R_{\mathrm{H}}
$$

and since $4 \pi R_{\mathrm{H}}=\alpha / r_{0} \quad$ we also get

$$
r_{\mathrm{e}}=\left(\frac{1}{137 \alpha}-1\right) r_{0}=1.391281 \times 10^{-14} \mathrm{~m} \quad \text { (electron radius) }
$$

Table 2. Parameters of $\mathrm{H}$ atom circular orbits up to the ionization, our results.

\begin{tabular}{cccccc}
\hline $\begin{array}{c}\text { Progressive } \\
\text { number } \\
\text { of each } \\
\text { circular } \\
\text { d-orbit n-th }\end{array}$ & $\begin{array}{c}\text { Total number } \\
\text { of admitted } \\
\text { photons along } \\
\text { each orbit } \\
n^{2}\end{array}$ & $\begin{array}{c}\text { Photons } \\
\text { effective } \\
\text { frequency } \\
\text { along orbit } r_{n} \\
v_{n}=v_{0} / n^{2}\end{array}$ & $\begin{array}{c}\text { Photons virtual } \\
\text { Frequency along } \\
\text { each orbit } r_{n}^{\prime} \\
v_{n}^{\prime}=v_{0}^{\prime} / n^{2}\end{array}$ & $\begin{array}{c}\text { Electron radial } \\
\text { speed due to } \\
n^{2} \text { photons } \\
w_{n^{2}}=2 h v_{0}^{\prime} / c m_{r}\end{array}$ & $\begin{array}{c}\text { Electron } \\
\text { orbital speed } \\
v_{0}^{\prime}=c / 137 \\
v_{n}^{\prime}=v_{0}^{\prime} / n\end{array}$ \\
\hline- & - & $\left(\times 10^{10} \mathrm{~Hz}\right)$ & $\left(\times 10^{10} \mathrm{~Hz}\right)$ & $\mathrm{m} / \mathrm{s}$ & $\mathrm{m} / \mathrm{s}$ \\
$1^{\text {st }}$ & 1 & $328,805.1$ & $328,977.9$ & $15,972.74$ & $2,188,266$ \\
$2^{\text {nd }}$ & 4 & $82,201.3$ & $82,244.4$ & $15,972.74$ & $1,094,133$ \\
$\ldots$ & $\ldots$ & $\ldots$ & $\ldots$ & $\ldots$ & $\ldots$ \\
137 th & $137^{2}$ & 17.52 & 17.53 & $15,972.74$ & $15,972.74$ \\
\hline
\end{tabular}




\subsection{Absorption/Emission Effect; Claimed Fall of Circling Electrons}

Referring to Figure 11 representing the Absorption of photons into a circling electron, let us assume the nucleus mass $m_{\mathrm{N}}>m_{\mathrm{e}}$ so to consider the nucleus fixed in the atom centre of gravity $B$.

Now, the expression of the total energy of the system photon-electron is given by

$$
T=E+U_{r}+K_{\mathrm{e}}+K_{r}
$$

where $E\left(=m c^{2}\right)$ is the energy of the incident light, $U_{r}\left(=-e^{2} / 4 \pi \varepsilon_{0} r\right)$ is the potential due to electrostatic attraction acting on the electron, $K_{\mathrm{e}}\left(=1 / 2 m_{\mathrm{e}} v^{2}\right)$ is the electron orbital kinetic energy, and $K_{r}\left(=1 / 2 m_{\mathrm{e}} W^{2}\right)$ its radial kinetic energy related to its radial speed $w$ due to the impact photon-electron.

Regarding the Absorption/Emission effect (elements on gaseous state), see Figure 11, along circular orbits it is $K_{r}=0$ and moreover, at the end of absorption, along the orbit $r_{2}$, (where the photons have been absorbed), it is $E_{2}=0$; hence between two circular orbits $r_{1}$ and $r_{2}$, the (101) gives

$$
E_{1}+U_{r 1}+K_{\mathrm{e} 1}=U_{r 2}+K_{\mathrm{e} 2} \text {. }
$$

Now, from (69) $U_{r}=-m_{\mathrm{e}} v^{2}$ and since $K_{\mathrm{e}}=1 / 2 m_{\mathrm{e}} v^{2}$, we get

$\left(U_{r}+K_{e}\right)=-1 / 2 m_{\mathrm{e}} v^{2}$ thus from (102) we get $E_{1}-1 / 2 m_{\mathrm{e}} v_{1}^{2}=-1 / 2 m_{\mathrm{e}} v_{2}^{2}$; so, as $E_{1}=$ $h v$, and since $m_{\mathrm{e}} v^{2}=e^{2} / 4 \pi \varepsilon_{0} r$ we find

$$
h v=1 / 2 m_{\mathrm{e}} v_{1}^{2}-1 / 2 m_{\mathrm{e}} v_{2}^{2}=\frac{e^{2}}{8 \pi \varepsilon_{0}}\left(\frac{1}{r_{1}}-\frac{1}{r_{2}}\right)
$$

Then, according to (71) we have $r_{1}=r_{0} n^{2}$ and $r_{2}=r_{0} k^{2}$ (with $k>n$ as $r_{2}>r_{1}$ ), thus

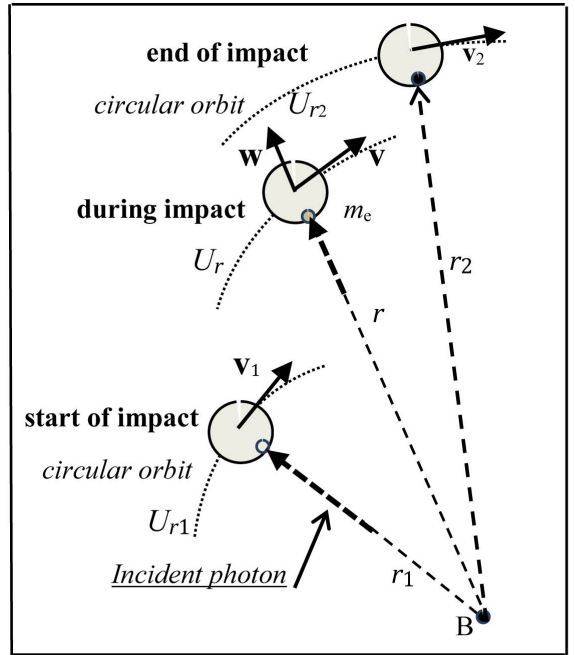

(a)

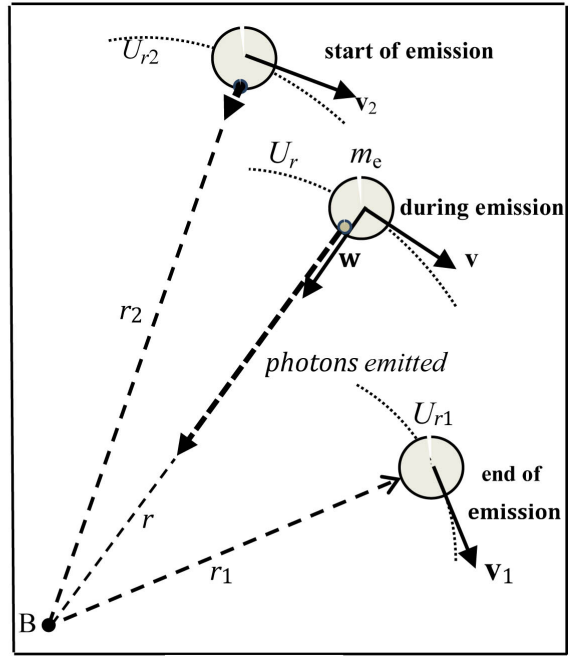

(b)

Figure 11. Absorption/Emission effect: (a) Incident photons are absorbed by the electron which moves toward wider orbits; (b) Emission: the electron moves toward inner orbits emitting photons. 


$$
v=\left(\frac{e^{2}}{8 \pi \varepsilon_{0} h r_{0}}\right)\left(\frac{1}{n^{2}}-\frac{1}{k^{2}}\right)
$$

and plugging the (74) written as $v_{0}=e^{2} / 8 \pi \varepsilon_{0} h r_{0}$, we find

$$
v=v_{0}\left(\frac{1}{n^{2}}-\frac{1}{k^{2}}\right)
$$

which is the photons frequency between two circular orbits, where $n$ represents the progressive specific number of each circular orbit and where $k$ turns out to have, for each of them, the values $k=n+1, n+2, \cdots, n_{i}$ which are, one by one, the number of the remaining external circular orbits, and where $n_{i}$ is the ionization orbit; on $\mathrm{H}$ atom, $n_{i}=137$.

Claimed fall of a circling electron into its nucleus: an electrical current emits an electro-magnetic radiation and therefore it is claimed that the circulating electrical charge of an electron should also emit an e.m. radiation yielding the electron, in a short time, to fall into the nucleus; but on our results, a free electron, moving, for instance, along a copper wire under an electrical potential difference, when entering into an atom influence, (at that moment the electron charge will return to face the atom nucleus), will release the necessary photons to reach the atom energy level corresponding to the energy previously received (during the absorption effect). Indeed, along circular orbits, it is $w=0$, therefore the absorption/emission of photons cannot happen along circular orbit, that is why the circling electrons are absorbing/emitting photons only between circular orbits, and therefore, the e.m. radiation is due to the emitted photons during their re-entry toward inner orbits; at this regard, the photons emission is necessary for the electrons not to fall into their nucleus.

\subsection{Photoelectric Effect: Number of Involved Photons}

Between the electron ground-state orbit $r_{0}$ and its extraction orbit $r \rightarrow \infty$ (intending on microscopic scale), the (101), valid for every interaction light-matter, gives

$$
E+U_{r 0}+K_{\mathrm{e} 0}+K_{r 0}=E^{\prime}+U_{r \rightarrow \infty}+K_{\mathrm{e} \infty}+K_{\mathrm{ae}}
$$

where $E^{\prime}$ is the energy of re-emitted light, $K_{\mathrm{ae}}=1 / 2 m_{\mathrm{e}} w_{\mathrm{ae}}^{2}$ the electron kinetic energy after its extraction (intending on macroscopic scale), $W_{\mathrm{ae}}$ its related radial speed, while the other terms have been defined referring to (101).

On ground-state, as also shown between Equations (102) and (103), it is

$$
U_{r 0}+K_{\mathrm{e} 0}=-m_{\mathrm{e}} v_{0}^{2}+1 / 2 m_{\mathrm{e}} v_{0}^{2}=-1 / 2 m_{\mathrm{e}} v_{0}^{2}=-W_{\mathrm{f}}
$$

with $v_{0}$ the electron speed along $r_{0}$ and $W_{\mathrm{f}}$ the work function (electron extraction work); now, at the start of impact it is $w=0$ giving $K_{r 0}\left(=1 / 2 m_{\mathrm{e}} w^{2}\right)=0$ while for $r \rightarrow \infty$, the electron orbital speed $v_{\infty} \rightarrow 0$,so

$$
\left(U_{r \rightarrow \infty}+K_{\mathrm{e} 0}\right)=-1 / 2 m_{\mathrm{e}} v_{\infty}^{2} \rightarrow 0
$$

hence the (106) gives

$$
E-W_{\mathrm{f}}=E^{\prime}+K_{\mathrm{ae}} \Rightarrow E=E^{\prime}+W_{\mathrm{f}}+K_{\mathrm{ae}}
$$


where $W_{\mathrm{f}}+K_{\mathrm{ae}}$ is the total kinetic energy transferred from light to electron, that is the sum of the sufficient energy to remove the electron plus the one to give it the energy after extraction.

On Photoelectric Effect $(\mathrm{PhE})$, the light scatters off an electron $\left(K_{\mathrm{ae}} \geq 0\right)$, but it is not re-emitted, hence $E^{\prime}=0$, so the (109), with $v_{\mathrm{f}}\left(=W_{\mathrm{f}} / h\right)$ the specific threshold frequency, becomes

$$
E=W_{\mathrm{f}}+K_{\mathrm{ae}} \Rightarrow h v=h v_{\mathrm{f}}+1 / 2 m_{\mathrm{e}} w_{\mathrm{ae}}^{2}
$$

showing that for $v=v_{\mathrm{f}}$ there is ionization with $w_{\mathrm{ae}}=0$.

At frequency $v_{\mathrm{f}}$ the electron radial speed $w_{\mathrm{f}}$ due to the impact of one photon, see Equation (85), is $w_{\mathrm{f}}=2 h v_{\mathrm{f}} / m_{\mathrm{e}} c=2 W_{\mathrm{f}} / m_{\mathrm{e}} c$, and writing the (66) as

$$
v_{0}=\left(2 h v_{\mathrm{f}} / m_{\mathrm{e}}\right)^{1 / 2}=\left(2 W_{\mathrm{f}} / m_{\mathrm{e}}\right)^{1 / 2}
$$

we get

$$
\frac{w_{\mathrm{f}}}{v_{0}}=\frac{\left(\frac{2 W_{\mathrm{f}}}{m_{\mathrm{e}} c}\right)}{\left(\frac{2 W_{\mathrm{f}}}{m_{\mathrm{e}}}\right)^{\frac{1}{2}}}=\left(\frac{2 W_{\mathrm{f}}}{m_{\mathrm{e}}}\right)^{1 / 2} / c=\frac{v_{0}}{c} \Rightarrow w_{\mathrm{f}}=\frac{v_{0}^{2}}{c}
$$

which is equal to (88), and since the values of $W_{\mathrm{f}}$ are in the range $\cong 2-6 \mathrm{eV}$, the Equation (112) gives $w_{\mathrm{f}} / v_{0} \cong 0.0028-0.0048$ meaning that the ionization (which for elements on solid state where electrons are circling along fixed orbits requires $w=v_{0}$ ), at frequency $v_{\mathrm{f}}$ needs $n_{\mathrm{f}}$ photons: indeed, the electron radial speed due to $n_{\mathrm{f}}$ photons with frequency $v_{\mathrm{f}}$, see (86), is

$$
w_{n \mathrm{f}}=n_{\mathrm{f}} w_{\mathrm{f}}=n_{\mathrm{f}} 2 W_{\mathrm{f}} / m_{\mathrm{e}} c
$$

so the ionization condition becomes $w_{n \mathrm{f}}=v_{0}$ leading to

$$
\begin{aligned}
& n_{\mathrm{f}} 2 W_{\mathrm{f}} / m_{\mathrm{e}} c=\left(2 W_{\mathrm{f}} / m_{\mathrm{e}}\right)^{1 / 2} \text { giving } \\
& n_{\mathrm{f}}=c / \sqrt{2 W_{\mathrm{f}} / m_{\mathrm{e}}}=c \sqrt{m_{\mathrm{e}} / 2 W_{\mathrm{f}}}=v_{0} / w_{\mathrm{f}}=c / v_{0}
\end{aligned}
$$

that is, on PhE, the number of photons at frequency $v_{\mathrm{f}}$ necessary for ionization $\left(w_{\mathrm{ae}}=0\right)$. Now, if $n_{\mathrm{f}}$ photons, at frequency $v_{\mathrm{f}}$, are sufficient for ionization, then the frequency

$$
n_{\mathrm{f}} v_{\mathrm{f}} \equiv v_{1}
$$

is sufficient for ionization ( $W_{\mathrm{ae}}=0$ ) with one photon only, so $v_{1}$ is the threshold between $\mathrm{PhE}$ and Compton effect which requires one photon only, as shown on next chapter.

To find the number $n_{1}$ related to the frequency $v_{1}$ let us equate the (66) written as $v_{0}=\sqrt{2 h v_{1} / m_{\mathrm{e}}}$, to the electron radial speed due to $n_{1}$ photons, $w_{n 1}=2 n h v_{1} / c m_{\mathrm{e}}$ yielding $\left(2 h v_{1} / m_{\mathrm{e}}\right)=\left(2 n_{1} h v_{1} / c m_{\mathrm{e}}\right)^{2}$ and then

$$
n_{1}^{2}=\frac{m_{\mathrm{e}} c^{2}}{2 h v_{1}}=\frac{m_{\mathrm{e}} c^{2}}{2 h n_{\mathrm{f}} v_{\mathrm{f}}}=\frac{m_{\mathrm{e}} c^{2}}{2 n_{\mathrm{f}} W_{\mathrm{f}}} \Rightarrow n_{1}=c \sqrt{m_{\mathrm{e}} / 2 n_{\mathrm{f}} W_{\mathrm{f}}}
$$

and plugging $W_{\mathrm{f}}=c^{2} m_{\mathrm{e}} / 2 n_{\mathrm{f}}^{2}$ as given by (114), we find

$$
n_{1}=\sqrt{n_{\mathrm{f}}} \text {. }
$$


For instance as for caesium (Cs), having $W_{\mathrm{f}} 1.95 \mathrm{eV}$, since $n_{\mathrm{f}}=c\left(m_{\mathrm{e}} / 2 W_{\mathrm{f}}\right)^{1 / 2} \cong 361.9$ we may infer $n_{\mathrm{f}}=361$ leading to $n_{1}=19$, while as for Be or Co, having $W_{\mathrm{f}} \cong 5 \mathrm{eV}$ we may infer $n_{\mathrm{f}}=225$ leading to $n_{1}=15$.

\subsection{Compton Effect and Its Equation via Doppler Effect}

Here, see Figure 12, the incident photon (length $\lambda$, frequency $v$ ), while ejecting a circling electron is also reflected $(\lambda, v)$ so the recoiling electron, emitting a photon $\lambda^{\prime}$ toward the Observer $\mathrm{A}$, represents a source in motion from A along the direction $\mathbf{w}$, implying an undoubted Doppler effect.

Now, on the basis that the incident photon starts to be reflected at the same time when it starts to hit the electron, and since $T^{\prime}=1 / v^{\prime}$ is the emission time of the reflected photon, it turns out that $T^{\prime}$ is also the whole interaction time (and this means that there is not a complete absorption of the incident photon followed by an emission). Now, with $T^{\prime}$ the whole impact time photon-electron, the momentum transferred from the incident light to the electron, as per (53), is

$$
p^{\prime}=m^{\prime} c=\gamma v^{\prime} c=\gamma c / T^{\prime}
$$

and the same value is then transferred from the reflected photon to the electron, so the Conservation of Momentum ( $\mathrm{CoM}$ ) along the direction normal to $\mathrm{w}$, becomes

$$
\gamma c / T^{\prime} \sin \theta=\gamma c / T \sin \theta^{\prime}
$$

giving $\theta=\theta^{\prime}$. Then, the length of the reflected photon, for the Observer A, see (17), is

$$
\lambda^{\prime}=\lambda+\Delta \lambda
$$

where $\Delta \lambda=w_{\mathrm{A}} T^{\prime}$, where $w_{\mathrm{A}}=w \cos \theta$ is the component of the electron speed along the direction of the Observer $\mathrm{A}$, and $T^{\prime}=1 / v^{\prime}$ is, for $\mathrm{A}$, the photon transit time, so we get

$$
\lambda^{\prime}-\lambda \equiv \Delta \lambda=w T^{\prime} \cos \theta
$$

Now the CoM along $\mathbf{w}$, being $\theta=\theta^{\prime}$, gives

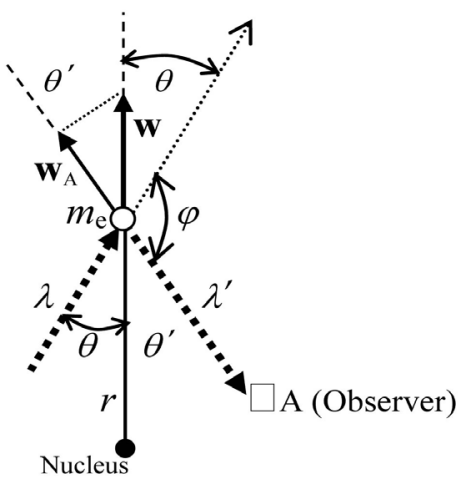

Figure 12. Compton effect (CE). $\varphi$ : angle between the direction of the incident photon and the scattered one $\left(\lambda^{\prime}\right) ; \theta$. angle between the direction of the incident photon and the recoiled electron. 


$$
\frac{\gamma c \cos \theta}{T^{\prime}}+\frac{\gamma c \cos \theta}{T^{\prime}}=m_{\mathrm{e}} w \Rightarrow w T^{\prime}=(2 \gamma c \cos \theta) / m_{\mathrm{e}}
$$

so the (121) becomes

$$
\lambda^{\prime}-\lambda(\equiv \Delta \lambda)=\frac{2 \gamma c \cos ^{2} \theta}{m_{\mathrm{e}}}=\frac{2 h \cos ^{2} \theta}{c m_{\mathrm{e}}}
$$

Now, $2 \theta+\varphi=\pi, \Rightarrow \theta=(\pi-\varphi) / 2$, hence $\cos \theta=\sin \varphi / 2$ and therefore

$$
\Delta \lambda=2 h \sin ^{2}\left(\frac{\varphi}{2}\right) / c m_{\mathrm{e}}
$$

and since $2 \sin ^{2}\left(\frac{\varphi}{2}\right)=(1-\cos \varphi)$, we get the Compton equation:

$$
\Delta \lambda=\lambda^{\prime}-\lambda=h(1-\cos \varphi) / \mathrm{cm}_{\mathrm{e}}
$$

which cannot be obtained via the Doppler effect relativistic equations regarding the light.

We point out that the (122) written as $w=\left(2 \gamma v^{\prime} c \cos \theta\right) / m_{\mathrm{e}}$, for $\cos \theta=1$ equals the (85), implying the impact of one photon only.

Now, the (123), for $\cos \theta=1$ gives $\lambda^{\prime}=\frac{c}{v}+\frac{2 \gamma c}{m_{\mathrm{e}}}$ we can write as:

$$
T^{\prime}=\frac{1}{v}+\frac{2 \gamma}{m_{\mathrm{e}}}=\left(m_{\mathrm{e}}+2 \gamma v\right) / v m_{\mathrm{e}}
$$

which plugged into (122) gives

$$
w=\left(\frac{2 \gamma c}{m_{\mathrm{e}}}\right) /\left(m_{\mathrm{e}}+2 \gamma v\right) / v m_{\mathrm{e}}=\frac{2 \gamma v c}{2 \gamma v+m_{\mathrm{e}}}=\frac{c}{1+m_{\mathrm{e}} / 2 \gamma v}
$$

yielding $w \rightarrow c$ for $v \rightarrow \infty$.

\section{Conclusions}

This paper is mainly based on three conditions: $c=u$ (with $u$ the total escape speed); a certain structure of the light (massive photons having variable length); the electron charge like a point-particle facing the atom nucleus during the electron orbit.

On these bases, we gave a classical reason to the apparent constancy of $c$, then we showed that the time dilation turns out to be an effect of the variation of the total gravitational potential $U$ (inducing a variation of $c$ ) and we also showed that the high gravitational red shifts are also due to the same reason; we also showed that the quantum numbers are nothing else than the numbers of photons admitted on free atoms along an integer number of electron orbits; then we gave a reason why the circling electrons cannot fall into their nucleus, and we also showed that the ionization of free atoms happens when the electron radial speed (due to the impact photons-electron) equals the electron orbital speed.

\section{Conflicts of Interest}

The authors declare no conflicts of interest regarding the publication of this paper. 


\section{References}

[1] Kragh, H. (1999) Cosmology and Controversy, 212.

[2] Gogberashvili, M., et al. (2014) Cosmological Parameter. ArXiv 1210.4618.

[3] Immerman, N. (2001) Nat'l Solar Observatory: The Universe. University of Massachusetts.

[4] Gott III, J.R., et al. The Astrophysics Journal, 624, 8.

[5] Van Dokkum, P. (2010) Nature, 468, 940-942. https://doi.org/10.1038/nature09578

[6] Schutz, B.F. (2003) Gravity from the Ground up. Cambridge University Press, 361.

[7] Blatt Frank, J. (1992) Modern Physics, Mossbauer Effect, McGraw Hill.

[8] Pound, R.V. and Rebka Jr., G.A. (1960) Physical Review Letters, 4, 337. https://doi.org/10.1103/PhysRevLett.4.337

[9] Pound, R.V. and Snider, J.L. (1964) Physical Review Letters, 13, 539. https://doi.org/10.1103/PhysRevLett.13.539

[10] Pound, R.V. and Snider, J.L. (1965) Physical Review, 140, 788. https://doi.org/10.1103/PhysRev.140.B788

[11] (Yearly) NASA Extragalactic Database: (i.e. Galaxy M86 Has $z \cong 0.001$ with $s \cong 16$ Mpc; M99 Has $z \cong+0.008$ with $s \cong 15 \mathrm{Mpc}$; NGC0063 Has $z \cong 0.004$ with $s \cong 20$ $\mathrm{Mpc}$; VCC0815 Has $z \cong 0.0025$ with $s \cong 20 \mathrm{Mpc}$ ).

[12] Halliday-Resnick (1981) Fundamentals of Physics, Chapter 47-2. 